\title{
Evaluation of CNN-based Single-Image Depth Estimation Methods
}

\author{
Tobias Koch $^{1} \quad$ Lukas Liebel $^{1} \quad$ Friedrich Fraundorfer $^{2,3} \quad$ Marco Körner $^{1}$ \\ ${ }^{1}$ Chair of Remote Sensing Technology, Computer Vision Research Group, Technical University of Munich \\ \{tobias.koch, lukas.liebel, marco.koerner\}@tum.de \\ ${ }^{2}$ Institute for Computer Graphics and Vision, Graz University of Technology \\ fraundorfer@icg.tugraz.at \\ ${ }^{3}$ Remote Sensing Technology Institute (IMF), German Aerospace Center (DLR)
}

\begin{abstract}
While an increasing interest in deep models for single-image depth estimation (SIDE) can be observed, established schemes for their evaluation are still limited. We propose a set of novel quality criteria, allowing for a more detailed analysis by focusing on specific characteristics of depth maps. In particular, we address the preservation of edges and planar regions, depth consistency, and absolute distance accuracy. In order to employ these metrics to evaluate and compare state-of-the-art SIDE approaches, we provide a new high-quality RGB-D dataset. We used a digital single-lens reflex (DSLR) camera together with a laser scanner to acquire high-resolution images and highly accurate depth maps. Experimental results show the validity of our proposed evaluation protocol.
\end{abstract}

\section{Introduction}

With the emergence of deep learning methods within the recent years and their massive influence on the computer vision domain, the problem of SIDE got addressed as well by many authors. These methods are in high demand for manifold scene understanding applications like, for instance, autonomous driving, robot navigation, or augmented reality systems. In order to replace or enhance traditional methods, convolutional neural network (CNN) architectures have been most commonly used and successfully shown to be able to infer geometrical information solely from presented monocular RGB or intensity images, as exemplary shown in Fig. 1. While these methods produce nicely intuitive results, proper evaluating the estimated depth maps is crucial for subsequent applications, e.g., their suitability for further 3D understanding scenarios [31]. Consistent and reliable relative depth estimates are, for instance, a key requirement for path planning approaches in robotics, augmented reality applications, or computational cinematography.

Nevertheless, the evaluation schemes and error metrics commonly used so far mainly consider the overall accuracy by reporting global statistics of depth residuals which does not give insight into the depth estimation quality at salient and important regions, like planar surfaces or geometric discontinuities. Hence, fairly reasonable reconstruction results, as shown in Fig. 1c, are probably positively evaluated, while still showing evident defects around edges. At the same time, the shortage of available datasets providing ground truth data of sufficient quality and quantity impedes precise evaluation. As this issue was reported by the authors of many recent SIDE papers, we aim at providing a new and extended evaluation scheme in order to overcome these deficiencies. In particular, as

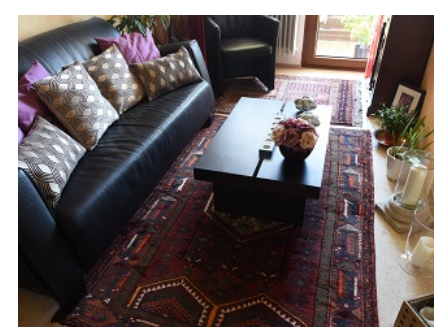

(a) RGB image

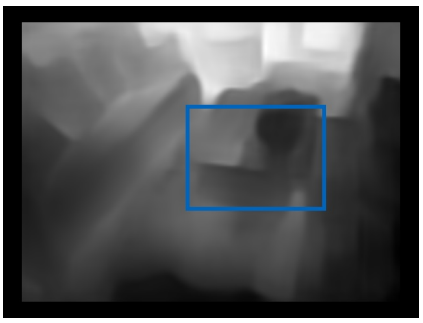

(c) Prediction

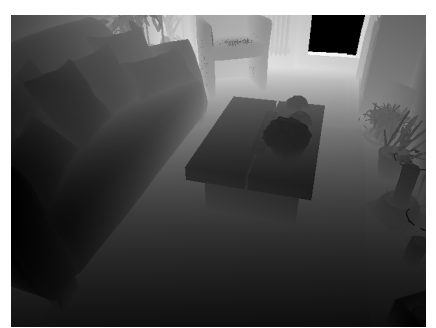

(b) Depth map

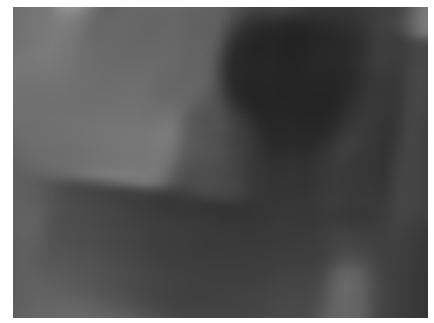

(d) Prediction detail
Figure 1: Sample image pair from our dataset and depth prediction using a state-of-the-art algorithm [6]. Although the quality of the depth map seems reasonable, the prediction suffers from artifacts, smoothing, missing objects, and inaccuracies in textured image regions

our main contributions, we $i$ ) present a new evaluation dataset acquired from diverse indoor scenarios containing high-resolution RGB images aside highly accurate depth maps from laser scans ${ }^{1}$, ii) introduce a set of new interpretable error metrics targeting the aforementioned issues, and iii) evaluate a variety of state-of-the-art methods using these data and performance measures.

${ }^{1}$ This dataset is freely available at www. $l m f$.bgu.tum. de/ibims1. 


\section{Related Work}

In this section, we introduce some of the most recent learningbased methods for predicting depth from a single image and review existing datasets used for training and evaluating the accuracy of these methods.

\subsection{Methods}

Most commonly, stereo reconstruction is performed from multiview setups, e.g., by triangulation of 3D points from corresponding 2D image points observed by distinct cameras (cf. multi-view stereo (MVS) or structure from motion (SfM) methods) [27]. Nevertheless, for already many decades, estimating depth or shape from monocular setups or single views is under scientific consideration [2] in psychovisual as well as computational research domains. Most prominently, methods based on the shape from shading (SfS) principle [12, 35] exploit intensity or color gradients from single images to predict geometrical structure of objects under the assumptions of homogeneous lighting and Lambertian surface properties. While these methods operate passively on single-shot images, active monocular alternatives have been investigated, such as, for instance, by exploiting the focus and defocus behavior $[8,30]$ or polarization cues [23, 13]. With the emergence of light field cameras, a further line of approaches [32,11] was developed. After several RGB-D datasets were released [10, 25, 28], data-driven learning-based approaches outperformed established modelbased methods. Especially deep learning-based methods have proven to be highly effective for this task and achieved current state-of-the-art results $[3,6,16,9,14,17,21,33,24,18,34,20]$. One of the first approaches using CNNs for regressing dense depth maps was presented by Eigen et al. [7] who employ two deep networks for first performing a coarse global prediction and refine the predictions locally afterwards. An extension to this approach uses deeper models and additionally predicts normals and semantic labels [6]. Liu et al. [21] combine CNNs and conditional random fields (CRFs) in a unified framework while making use of superpixels for preserving sharp edges. Laina et al. [16] tackle this problem with a fully convolutional network consisting of a feature map up-sampling within the network. While Li et al. [18] employ a novel set loss and a two-streamed $\mathrm{CNN}$ that fuses predictions of depth and depth gradients, $\mathrm{Xu}$ et al. [34] propose to integrate complementary information derived from multiple CNN side outputs using CRFs.

\subsection{Existing Benchmark Datasets}

In order to evaluate SIDE methods, any dataset containing corresponding RGB and depth images can be considered, which also comprises benchmarks originally designed for the evaluation of MVS approaches. Strecha et al. [29] propose a MVS benchmark providing overlapping images with camera poses for six different outdoor scenes and a ground truth point cloud obtained by a laser scanner. More recently, two MVS benchmarks, the ETH3D [26] and the Tanks \& Temples [15] datasets, have been released. Although these MVS benchmarks contain high resolution images and accurate ground truth data obtained from laser scanners, the setups are not designed for SIDE methods. Usually, a scene is scanned from multiple aligned laser scans and image sequences are acquired of the same scene. However, it cannot be guaranteed that the corresponding depth images are dense and could, therefore, exhibit gaps in the depth maps due to occlusions. Despite the possibility of acquiring a large number of image pairs, they mostly comprise only a limited scene variety and are highly redundant due to the image overlap. Currently, SIDE methods are tested on mainly three different datasets. Make3D [25], as one example, contains 534 outdoor images and aligned depth maps acquired from a custom-built 3D scanner, but suffers from a very low resolution of the depth maps and a rather limited scene variety. The Kitti dataset [10] contains street scenes captured out of a moving car. The dataset contains RGB images together with depth maps from a Velodyne laser scanner. However, depth maps are only provided in a very low resolution which furthermore suffer from irregularly and sparsely spaced points. The most frequently used dataset is the NYU depth v2 dataset [28] containing 464 indoor scenes with aligned RGB and depth images from video sequences obtained from a Microsoft Kinect v1 sensor. A subset of this dataset is mostly used for training deep networks, while another 654 image and depth pairs serve for evaluation. This large number of image pairs and the various indoor scenarios facilitated the fast progress of SIDE methods. However, active RGB-D sensors, like the Kinect, suffer from a short operational range, occlusions, gaps, and erroneous specular surfaces. The recently released Matterport 3D [4] dataset provides an even larger amount of indoor scenes collected from a custom-built 3D scanner consisting of three RGB-D cameras. This dataset is a valuable addition to the NYU-V2 but also suffers from the same weaknesses of active RGB-D sensors. A comparison of our proposed dataset and the existing datasets for evaluating SIDE methods is provided in Table 1.

\section{Error Metrics}

This section describes established metrics and our new proposed ones allowing for a more detailed analysis.

\subsection{Commonly Used Error Metrics}

Established error metrics consider global statistics between a predicted depth map $\boldsymbol{Y}$ and its ground truth depth image $\boldsymbol{Y}^{*}$ with $T$ depth pixels. Beside visual inspections of depth maps or projected 3D point clouds, the following error metrics are exclusively used in all relevant recent publications $[7,6,16,19$, 34]:

Threshold: \% of $y$ such that $\max \left(\frac{y_{i}}{y_{i}^{*}}, \frac{y_{i}^{*}}{y_{i}}\right)=\sigma<t h r$

Absolute rel. diff.: $\operatorname{rel}=\frac{1}{T} \sum_{i, j}\left|y_{i, j}-y_{i, j}^{*}\right| / y_{i, j}^{*}$

Squared rel. diff.: srel $=\frac{1}{T} \sum_{i, j}\left|y_{i, j}-y_{i, j}^{*}\right|^{2} / y_{i, j}^{*}$

RMS (linear): RMS $=\sqrt{\frac{1}{T} \sum_{i, j}\left|y_{i, j}-y_{i, j}^{*}\right|^{2}}$

RMS (log): $\log _{10}=\sqrt{\frac{1}{T} \sum_{i, j}\left|\log y_{i, j}-\log y_{i, j}^{*}\right|^{2}}$ 
Table 1: Comparison of existing datasets related to SIDE evaluation with respect to different dataset characteristics. Higher resolutions are specified in brackets, if available in the datasets

\begin{tabular}{|c|c|c|c|c|c|}
\hline Benchmark & Setting & Sensor & $\begin{array}{l}\text { Resolution } \\
\text { (in } \mathrm{Mpx} \text { ) }\end{array}$ & Scenes & Images \\
\hline Strecha [29] & Outdoor & LiDAR & 6 & 6 & 30 \\
\hline ETH3D [26] & Various & LiDAR & $0.4(24)$ & 25 & 898 \\
\hline Tanks \& Temples [15] & Various & LiDAR & 2 & 14 & $150 \mathrm{k}$ \\
\hline Make3D [25] & Outdoor & LiDAR & 0.017 & - & 534 \\
\hline Kitti [10] & Street & LiDAR & 0.5 & - & 697 \\
\hline NYU-v2 [28] & Indoor & RGB-D & 0.3 & 464 & 654 \\
\hline Matterport 3D [4] & Indoor & RGB-D & 0.8 & 90 & $200 \mathrm{k}$ \\
\hline IBims-1 (proposed) & Indoor & LiDAR & $0.3(6)$ & 20 & 54 \\
\hline
\end{tabular}

Even though these statistics are good indicators for the general quality of predicted depth maps, they could be delusive. Particularly, the standard metrics are not able to directly assess the planarity of planar surfaces or the correctness of estimated plane orientations. Furthermore, it is of high relevance that depth discontinuities are precisely located, which is not reflected by the standard metrics.

\subsection{Proposed Error Metrics}

In order to allow for a more meaningful analysis of predicted depth maps and a more complete comparison of different algorithms, we present a set of new quality measures that specify on different characteristics of depth maps which are crucial for many applications. These are meant to be used in addition to the traditional error metrics introduced in Section 3.1. When talking about depth maps, the following questions arise that should be addressed by our new metrics: How is the quality of predicted depth maps for different absolute scene depths? Can planar surfaces be reconstructed correctly? Can all depth discontinuities be represented? How accurately are they localized? Are depth estimates consistent over the whole image area?

\subsubsection{Distance-Related Assessment}

Established global statistics are calculated over the full range of depth comprised by the image and therefore do not consider different accuracies for specific absolute scene ranges. Hence, applying the standard metrics for specific range intervals by discretizing existing depth ranges into discrete bins (e.g., onemeter depth slices) allows investigating the performance of predicted depths for close and far ranged objects independently.

\subsubsection{Planarity}

Man-made objects, in particular, can often be characterized by planar structures like walls, floors, ceilings, openings, and diverse types of furniture. However, global statistics do not directly give information about the shape correctness of objects within the scene. Predicting depths for planar objects is challenging for many reasons. Primarily, these objects tend to lack texture and only differ by smooth color gradients in the image, from which it is hard to estimate the correct orientation of a
3D plane with three-degrees-of-freedom. In the presence of textured planar surfaces, it is even more challenging for a SIDE approach to distinguish between a real depth discontinuity and a textured planar surface, e.g., a painting on a wall. As most methods are trained on large indoor scenes, like NYU-V2, a correct representation of planar structures is an important task for SIDE, but can hardly be evaluated using established standard metrics. For this reason, we propose to use a set of annotated images defining various planar surfaces $\boldsymbol{\pi}_{k}^{*}=\left(\boldsymbol{\eta}_{k}^{*}, d_{k}^{*}\right)$ (walls, table surfaces, and floors) and evaluate the flatness and orientation of predicted 3D planes $\boldsymbol{\pi}_{k}$ compared to ground truth 3D planes $\boldsymbol{\pi}_{k}^{*}$. In particular, a masked depth map $\boldsymbol{Y}_{k}$ of a particular planar surface is projected to 3D points $\boldsymbol{P}_{k ; i, j}$ where 3D planes $\boldsymbol{\pi}_{k}$ are robustly fitted to both the ground truth and predicted 3D point clouds $\mathcal{P}_{k}^{*}=\left\{\boldsymbol{P}_{k ; i, j}^{*}\right\}_{i, j}$ and $\mathcal{P}_{k}=\left\{\boldsymbol{P}_{k ; i, j}\right\}_{i, j}$, respectively. The planarity error

$$
\varepsilon_{\mathrm{PE}}^{\mathrm{plan}}\left(\boldsymbol{Y}_{k}\right)=\mathbb{V}\left[\sum_{\boldsymbol{P}_{k ; i, j} \in \mathcal{P}_{k}} d\left(\boldsymbol{\pi}_{k}, \boldsymbol{P}_{k ; i, j}\right)\right]
$$

is then quantified by the standard deviation of the averaged distances between the predicted 3D point cloud and its corresponding 3D plane estimate. The orientation error

$$
\varepsilon_{\mathrm{PE}}^{\text {orie }}\left(\boldsymbol{Y}_{k}\right)=\operatorname{acos}\left(\boldsymbol{\eta}_{k}^{\top} \cdot \boldsymbol{\eta}_{k}^{*}\right)
$$

is defined as the $3 \mathrm{D}$ angle difference between the normal vectors of predicted and ground truth 3D planes. Figs. $2 \mathrm{a}$ and $2 \mathrm{~b}$ illustrate the proposed planarity errors. Note that the predicted depth maps are scaled w.r.t. the ground truth depth map, in order to eliminate scaling differences of compared methods.

\subsubsection{Location Accuracy of Depth Boundaries}

Beside planar surfaces, captured scenes, especially indoor scenes, cover a large variety of scene depths caused by any object in the scene. Depth discontinuities between two objects are represented as strong gradient changes in the depth maps. In this context, it is important to examine whether predicted depths maps are able to represent all relevant depth discontinuities in an accurate way or if they even create fictitious depth discontinuities confused by texture. An analysis of depth discontinuities can be best expressed by detecting and comparing 


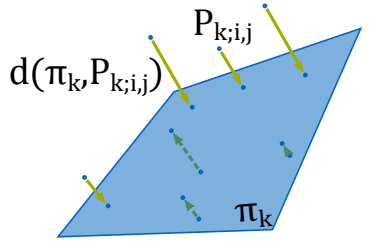

(a) $\varepsilon_{\mathrm{PE}}^{\text {plan }}$

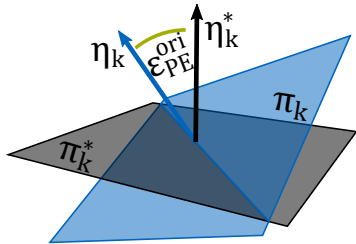

(b) $\varepsilon_{\mathrm{PE}}^{\text {orie }}$

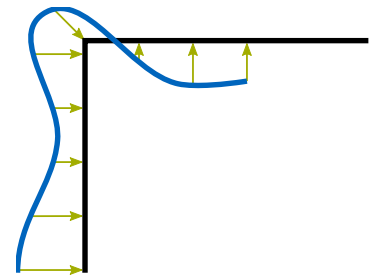

(c) $\varepsilon_{\mathrm{DBE}}^{\mathrm{acc}}$

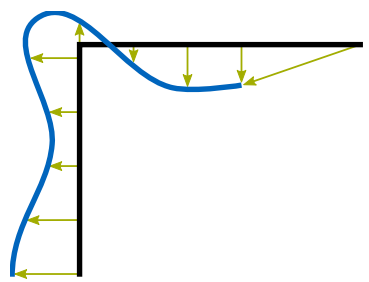

(d) $\varepsilon_{\mathrm{DBE}}^{\mathrm{comp}}$

Figure 2: Visualizations of the proposed error metrics for planarity errors ( $\mathrm{a}$ and $\mathrm{b}$ ) and depth boundary errors (c and d)

edges in predicted and ground truth depth maps. Location accuracy and sharp edges are of high importance for generating a set of ground truth depth transitions which cannot be guaranteed by existing datasets acquired from RGB-D sensors. Ground truth edges are extracted from our dataset by first generating a set of tentative edge hypotheses using structured edges [5] and then manually selecting important and distinct edges subsequently. In order to evaluate predicted depth maps, edges $\boldsymbol{Y}_{\text {bin }}$ are extracted using structured edges and compared to the ground truth edges $\boldsymbol{Y}_{\text {bin }}^{*}$ via truncated chamfer distance of the binary edge images. Specifically, an Euclidean distance transform is applied to the ground truth edge image $\boldsymbol{E}^{*}=D T\left(\boldsymbol{Y}_{\text {bin }}^{*}\right)$, while distances exceeding a given threshold $\theta$ are ignored. We define the depth boundary errors (DBEs), comprised of an accuracy measure

$$
\varepsilon_{\mathrm{DBE}}^{\mathrm{acc}}(\boldsymbol{Y})=\frac{1}{\sum_{i} \sum_{j} y_{\mathrm{bin} ; i, j}} \sum_{i} \sum_{j} e_{i, j}^{*} \cdot y_{\mathrm{bin} ; i, j}
$$

by multiplying the predicted binary edge map with the distance map and a subsequent accumulation of the pixel distances towards the ground truth edge. As this measure does not consider any missing edges in the predicted depth image, we also define a completeness error

$$
\varepsilon_{\mathrm{DBE}}^{\mathrm{comp}}(\boldsymbol{Y})=\frac{1}{\sum_{i} \sum_{j} y_{\mathrm{bin} ; i, j}^{*}} \sum_{i} \sum_{j} e_{i, j} \cdot y_{\mathrm{bin} ; i, j}^{*}
$$

by accumulating the ground truth edges multiplied with the distance image of the predicted edges $\boldsymbol{E}=D T\left(\boldsymbol{Y}_{\text {bin }}\right)$. A visual explanation of the DBEs are illustrated in Figs. 2c and 2d.

\subsubsection{Directed Depth Error}

For many applications, it is of high interest that depth images are consistent over the whole image area. Although the absolute depth error and squared depth error give information about the correctness between predicted and ground truth depths, they do not provide information if the predicted depth is estimated too short or too far. For this purpose, we define the directed depth errors (DDEs)

$$
\begin{aligned}
& \varepsilon_{\mathrm{DDE}}^{+}(\boldsymbol{Y})=\frac{\left|\left\{y_{i, j} \mid d_{\mathrm{sgn}}\left(\boldsymbol{\pi}, \boldsymbol{P}_{i, j}\right)>0 \wedge d_{\mathrm{sgn}}\left(\boldsymbol{\pi}, \boldsymbol{P}_{i, j}^{*}\right)<0\right\}\right|}{T} \\
& \varepsilon_{\mathrm{DDE}}^{-}(\boldsymbol{Y})=\frac{\left|\left\{y_{i, j} \mid d_{\mathrm{sgn}}\left(\boldsymbol{\pi}, \boldsymbol{P}_{i, j}\right)<0 \wedge d_{\mathrm{sgn}}\left(\boldsymbol{\pi}, \boldsymbol{P}_{i, j}^{*}\right)>0\right\}\right|}{T}
\end{aligned}
$$

as the proportions of too far and too close predicted depth pixels $\varepsilon_{\mathrm{DDE}}^{+}$and $\varepsilon_{\mathrm{DDE}}^{-}$. In practice, a reference depth plane $\pi$ is defined at a certain distance (e.g., at $3 \mathrm{~m}, c f$. Fig. 7c) and all predicted depths pixels which lie in front and behind this plane are masked and assessed according to their correctness using the reference depth images.

\section{Dataset}

As described in the previous sections, our proposed metrics require extended ground truth which is not yet available in standard datasets. Hence, we compiled a new dataset according to these specifications.

\subsection{Acquisition}

For creating such a reference dataset, high-quality optical RGB images and depth maps had to be acquired. Practical considerations included the choice of suitable instruments for the acquisition of both parts. Furthermore, a protocol to calibrate both instruments, such that image and depth map align with each other, had to be developed. An exhaustive analysis and comparison of different sensors considered for the data acquisition can be found in the supplementary material. This comparison clearly shows the advantages of using a laser scanner and a DSLR camera compared to active sensors like RGB-D cameras or passive stereo camera rigs. We therefore used the respective setup for the creation of our dataset.

In order to record the ground truth for our dataset, we used a highly accurate Leica HDS7000 laser scanner, which stands out for high point cloud density and very low noise level. We acquired the scans with $3 \mathrm{~mm}$ point spacing and $0.4 \mathrm{~mm}$ RMS at $10 \mathrm{~m}$ distance. As our laser scanner does not provide RGB images along with the point clouds, an additional camera was used in order to capture optical imagery. The usage of a reasonably high-quality camera sensor and lens allows for capturing images in high resolution with only slight distortions and a high stability regarding the intrinsic parameters. For the experiments, we chose and calibrated a Nikon D5500 DSLR camera and a Nikon AF-S Nikkor 18-105 mm lens, mechanically fixed to a focal length of approximately $18 \mathrm{~mm}$.

Using our sensor setup, synchronous acquisition of point clouds and RGB imagery is not possible. In order to acquire depth maps without parallax effects, the camera was mounted on a custom panoramic tripod head which allows to freely position 
the camera along all six degrees of freedom. This setup can be interchanged with the laser scanner, ensuring coincidence of the optical center of the camera and the origin of the laser scanner coordinate system after a prior calibration of the system. A visualization of our acquisition setup can be found in the supplementary material. It is worth noting, that every single RGB-D image pair of our dataset was obtained by an individual scan and image capture with the aforementioned strategy in order to achieve dense depth maps without gaps due to occlusions.

\subsection{Registration and Processing}

The acquired images were undistorted using the intrinsic camera parameters obtained from the calibration process. In order to register the camera towards the local coordinate system of the laser scanner, we manually selected a sufficient number of corresponding 2D and 3D points and estimated the camera pose using EPnP [22]. This registration of the camera relative to the point cloud yielded only a minor translation, thanks to the pre-calibrated platform. Using this procedure, we determined the 6D pose of a virtual depth sensor which we use to derive a matching depth map from the 3D point cloud. In order to obtain a depth value for each pixel in the image, the images were sampled down to two different resolutions. We provide a high-quality version with a resolution of $3000 \times 2000$ px and a cropped NYU-v2-like version with a resolution of $640 \times 480 \mathrm{px}$. 3D points were projected to a virtual sensor with the respective resolution. For each pixel, a depth value was calculated, representing the depth value of the $3 \mathrm{D}$ point with the shortest distance to the virtual sensor. It is worth highlighting that depth maps were derived from the 3D point cloud for both versions of the images separately. Hence, no down-sampling artifacts are introduced for the lower-resolution version. The depth maps for both, the high-quality and the NYU-V2-like version, are provided along with the respective images.

\subsection{Contents}

Following the described procedure, we compiled a dataset, which we henceforth refer to as the independent benchmark images and matched scans v1 (IBims-1) dataset. The dataset is mainly composed of reference data for the direct evaluation of depth maps, as produced by SIDE methods. As described in the previous sections, pairs of images and depth maps were acquired and are provided in two different versions, namely a high-quality version and a NYU-v2-like version. Example pairs of images and matching depth maps from IBims-1 are shown in Figs. 1a and $1 \mathrm{~b}$ and Figs. $3 \mathrm{a}$ and $3 \mathrm{~b}$, respectively. More samples of different scenes are provided in the supplementary material.

Additionally, several manually created masks are provided. Examples for all types of masks are shown in Fig. 3c, while statistics of the plane annotations are listed in Table 2.

In order to allow for evaluation following the proposed DBE metric, we provide distinct edges for all images. Edges have been detected automatically and manually selected. Fig. 3d

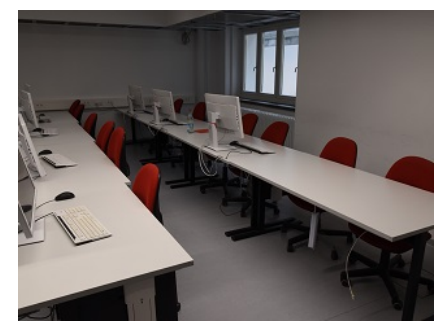

(a) Camera image

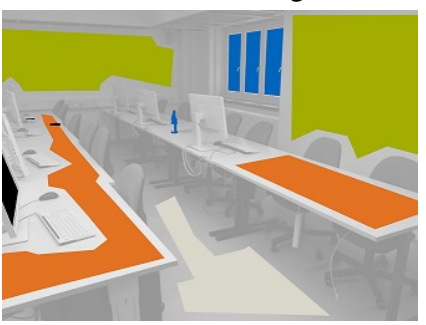

(c) Masks

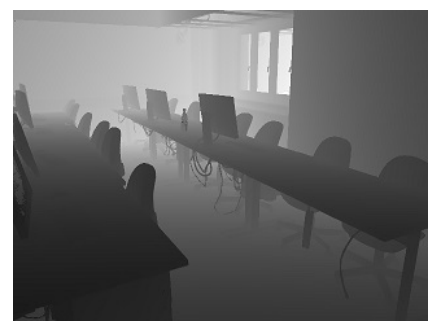

(b) Ground truth

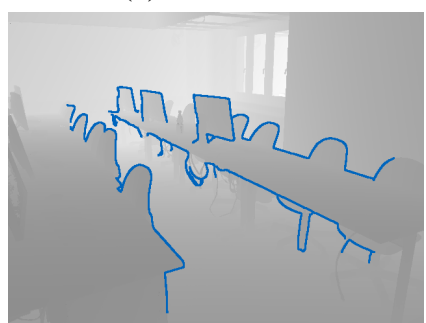

(d) Distinct edges
Figure 3: Sample from the main part of the proposed IBims-1 dataset with (a) RGB image, (b) depth map, (c) several masks with semantic annotations (i.e., walls ( $\square$ ), floor ( $\square$ ), tables ( $\square$ ), transparent objects ( $\square$ ), and invalid pixels ( $)$ ), and (d) distinct edges ( $(-)$

Table 2: Number and statistics of manually labeled plane masks in IBims -1

\begin{tabular}{|c|c|c|c|}
\hline Plane Type & Images & Instances & Pixels (for NYUv2 res.) \\
\hline Floor & 15 & 17 & 633729 \\
\hline Table & 21 & 28 & 106428 \\
\hline Wall & 34 & 71 & 1955804 \\
\hline
\end{tabular}

shows an example for one of the scenes from IBims-1.

This main part of the dataset contains 54 scenes in total ${ }^{2}$. So far, the NYU-V2 dataset is still the most comprehensive and accurate indoor dataset for training data-demanding deep learning methods. Since this dataset has most commonly been used for training the considered SIDE methods, IBims-1 is designed to contain similar scenarios. Our acquired scenarios include various indoor settings, such as office, lecture, and living rooms, computer labs, a factory room, as well as more challenging ones, such as long corridors and potted plants. A comparison regarding the scene variety between NYU-V2 and IBims-1 can be seen in Fig. 4b. Furthermore, IBims-1 features statistics comparable to NYU-V2, such as the distribution of depth values, shown in Fig. 4a, and a comparable field of view.

Additionally, four sets of auxiliary, more specific and especially challenging images are provided. These depict four outdoor images containing cars, buildings and far ranges. The second set contains special cases which are expected to mislead SIDE methods. These show printed samples from the NYU-V2 dataset and printed black and white patterns from the Pattern dataset [1] hung on a wall. Those could potentially give valuable insights, as they reveal what kind of image features SIDE methods exploit. Fig. 8a shows examples from both categories. No depth maps are provided for those images, as the region

\footnotetext{
${ }^{2}$ The final dataset will contain 100 image pairs
} 


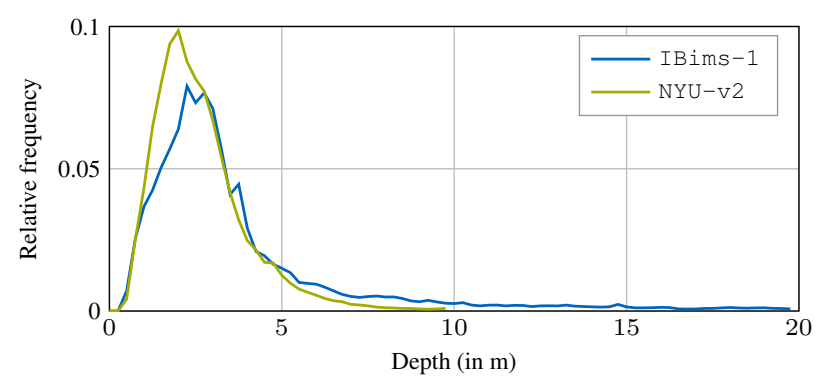

(a) Distribution of depth values

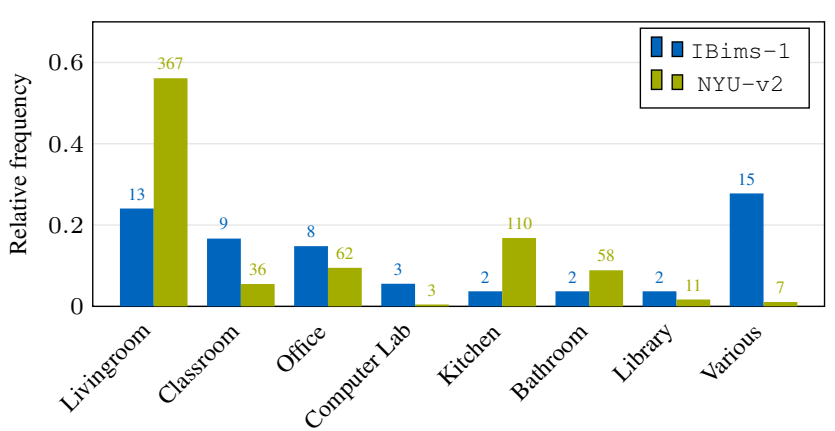

(b) Distribution of samples for each scene type. Absolute numbers are given above

Figure 4: IBims-1 dataset statistics compared to the NYU-v2 dataset. Distribution of depth values (a) and scene variety (b)

of interest is supposed to be approximately planar and depth estimates are, thus, easy to assess qualitatively. The third set of auxiliary images contains geometrically and radiometrically augmented IBims-1 images to test the robustness of SIDE methods. The last set comprises additional handheld images for every scene with viewpoint changes towards the reference images allowing to validate multi-view stereo algorithms with high-quality ground truth depth maps.

\section{Evaluation}

In this section, we evaluate the quality of existing SIDE methods using both established and proposed metrics for our reference test dataset, as well as for the commonly used NYU-v2 dataset. Furthermore, additional experiments were conducted to investigate the general behavior of SIDE methods, i.e., the robustness of predicted depth maps to geometrical and color transformations and the planarity of textured vertical surfaces. For evaluation, we compared several state-of-the-art methods, namely those proposed by Eigen and Fergus [7], Eigen et al. [6], Liu et al. [20], Laina et al. [16], and Li et al. [19]. It is worth mentioning that all of these methods were solely trained on the NYU-v2 dataset. Therefore, differences in the results are expected to arise from the developed methodology rather than the training data.

\subsection{Evaluation Using Proposed Metrics}

In the following, we report the results of evaluating SIDE methods on both NYU-v2 and IBims-1 using our newly proposed metrics. Please note, that due to the page limit, only few graphical results can be displayed in the following sections. Please refer to the supplementary material to find elaborate results for all evaluations.

\subsubsection{Distance-Related Assessment}

The results of evaluation using commonly used metrics on NYU-v2 and IBims-1 unveil lower overall scores for our dataset (please refer to Table 3 for IBims-1 and the supplementary material for $\mathrm{NYU}-\mathrm{v} 2$ ). In order to get a better understanding of these results, we evaluated the considered methods
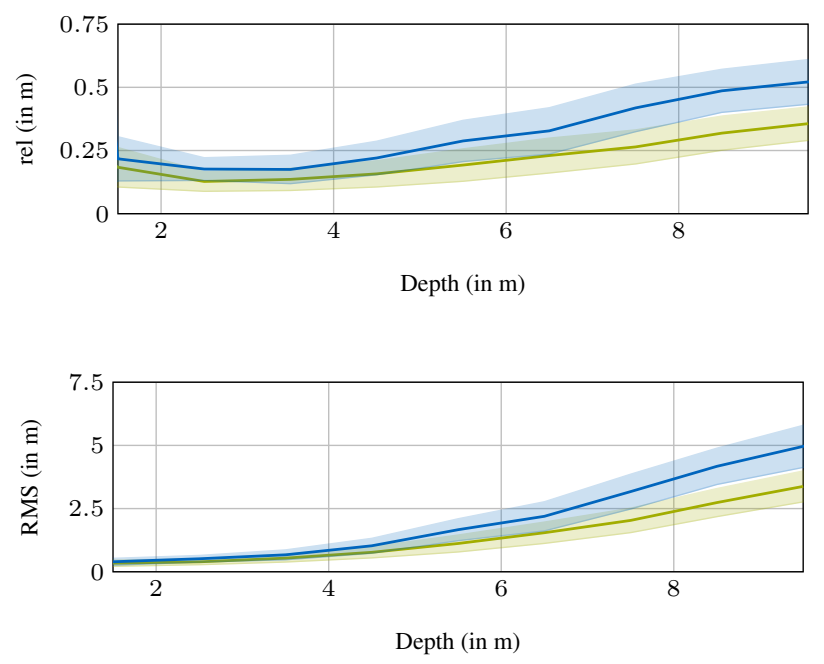

Figure 5: Distance-related global errors (top: relative error and bottom: RMS) for NYU-v2 (mean: —_ \pm 0.5 std: ) and IBims-1 (mean: $\pm 0.5 \mathrm{std}$ ) using the method of Li et al. [19]

on specific range intervals, which we set to $1 \mathrm{~m}$ in our experiments. Fig. 5 shows the error band of the relative and RMS errors of the method proposed by Li et al. [19] applied to both datasets. The result clearly shows a comparable trend on both datasets for the shared depth range. This proves our first assumption, that the overall lower scores originate from the huge differences at depth values beyond the $10 \mathrm{~m}$ depth range. On the other hand, the results reveal the generalization capabilities of the networks, which achieve similar results on images from another camera with different intrinsics and for different scenarios. It should be noted that the error bands, which show similar characteristics for different methods and error metrics, correlate with the depth distributions of the datasets, shown in Fig. 4a.

\subsubsection{Planarity}

To investigate the quality of reconstructed planar structures, we evaluated the different methods with the planarity and orientation errors $\varepsilon_{\mathrm{PE}}^{\mathrm{plan}}$ and $\varepsilon_{\mathrm{PE}}^{\text {orie }}$, respectively, as defined in Section 3.2.2, for different planar objects. In particular, we distinguished between horizontal and vertical planes and used masks 
Table 3: Quantitative results for standard metrics and proposed PE, DBE, and DDE metrics on IBims-1 applying different SIDE methods

\begin{tabular}{|c|c|c|c|c|c|c|c|c|c|c|c|c|c|}
\hline \multirow[t]{2}{*}{ Method } & \multicolumn{6}{|c|}{ Standard Metrics $\left(\sigma_{i}=1.25^{i}\right)$} & \multicolumn{2}{|c|}{$\mathrm{PE}\left(\right.$ in $\left.\mathrm{m} /{ }^{\circ}\right)$} & \multicolumn{2}{|c|}{ DBE (in px) } & \multicolumn{3}{|c|}{ DDE (in \%) } \\
\hline & rel & $\log _{10}$ & RMS & $\sigma_{1}$ & $\sigma_{2}$ & $\sigma_{3}$ & $\varepsilon_{\mathrm{PE}}^{\text {plan }}$ & $\varepsilon_{\mathrm{PE}}^{\text {orie }}$ & $\varepsilon_{\mathrm{DBE}}^{\mathrm{acc}}$ & $\varepsilon_{\mathrm{DBE}}^{\mathrm{comp}}$ & $\varepsilon_{\mathrm{DDE}}^{0}$ & $\varepsilon_{\mathrm{DDE}}^{-}$ & $\varepsilon_{\mathrm{DDE}}^{+}$ \\
\hline Eigen [7] & 0.36 & 0.22 & 2.92 & 0.35 & 0.63 & 0.79 & 0.18 & 33.27 & 3.60 & 48.08 & 64.53 & 32.31 & 3.15 \\
\hline Eigen (AlexNet) [6] & 0.32 & 0.18 & 2.63 & 0.42 & 0.72 & 0.82 & 0.21 & 26.64 & 3.01 & 32.00 & 74.65 & 21.51 & 3.84 \\
\hline Eigen (VGG) [6] & 0.29 & 0.17 & 2.59 & 0.47 & 0.73 & 0.85 & 0.17 & 21.64 & 3.16 & 27.47 & 75.10 & 23.44 & 1.46 \\
\hline Laina [16] & 0.27 & 0.16 & 2.42 & 0.56 & 0.76 & 0.84 & 0.22 & 32.02 & 4.58 & 38.41 & 77.12 & 20.89 & 1.99 \\
\hline Liu [20] & 0.33 & 0.17 & 2.51 & 0.46 & 0.73 & 0.84 & 0.22 & 31.90 & 2.32 & 16.85 & 77.27 & 16.38 & 6.35 \\
\hline $\mathrm{Li}[19]$ & 0.25 & 0.14 & 2.32 & 0.58 & 0.79 & 0.86 & 0.20 & 26.67 & 2.36 & 21.02 & 80.99 & 16.44 & 2.57 \\
\hline
\end{tabular}
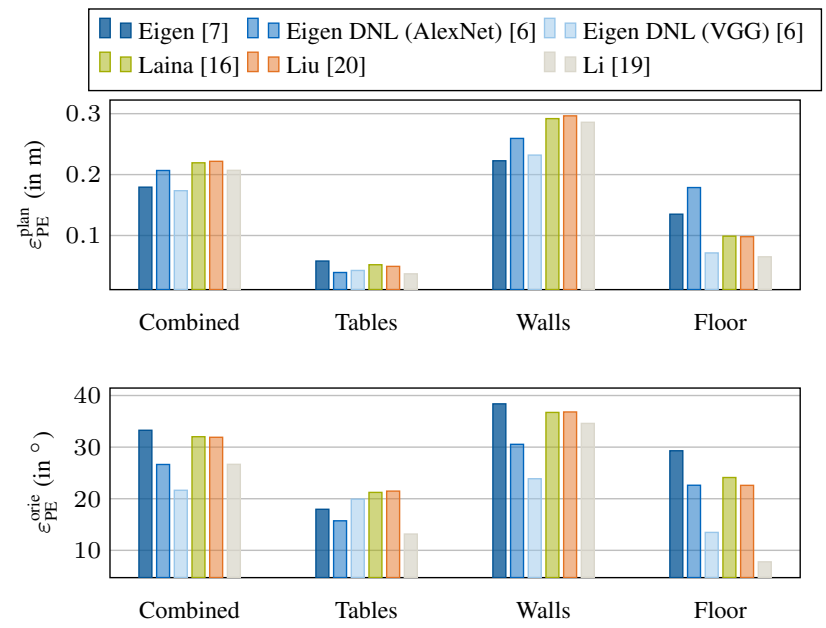

Figure 6: Results for the planarity metrics $\varepsilon_{\mathrm{PE}}^{\mathrm{plan}}$ (top) and $\varepsilon_{\mathrm{PE}}^{\text {orie }}$ (bottom) on IBims -1

from our dataset. Fig. 6 and Table 3 show the results for the IBims-1 dataset. Beside a combined error, including all planar labels, we separately computed the errors for the individual objects as well. The results show different performances for individual classes, especially orientations of floors were predicted in a significantly higher accuracy for all methods, while the absolute orientation error for walls is surprisingly high. Apart from the general performance of all methods, substantial differences between the considered methods can be determined. It is notable that the method of $\mathrm{Li}$ et al. [19] achieved much better results in predicting orientations of horizontal planes but also performed rather bad on vertical surfaces.

\subsubsection{Location Accuracy of Depth Boundaries}

The high quality of our reference dataset facilitates an accurate assessment of predicted depth discontinuities. As ground truth edges, we used the provided edge maps from our dataset and computed the accuracy and completeness errors $\varepsilon_{\mathrm{DBE}}^{\mathrm{acc}}$ and $\varepsilon_{\mathrm{DBE}}^{\mathrm{comp}}$, respectively, introduced in Section 3.2.3. Quantitative results for all methods are listed in Table 3. Comparing the accuracy error of all methods, Liu et al. [20] and Li et al. [19] achieved best results in preserving true depth boundaries, while other methods tended to produce smooth edges loosing sharp transitions which can be seen in Figs. 7a and 7b. This smoothing property also affected the completeness error, resulting in missing edges expressed by larger values for $\varepsilon_{\mathrm{DBE}}^{\mathrm{comp}}$.

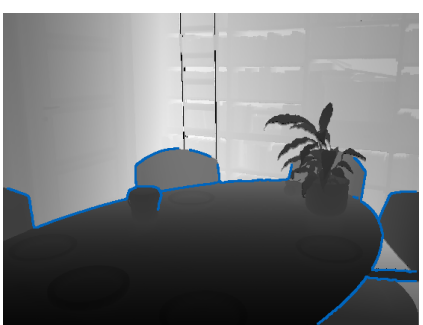

(a) Ground truth

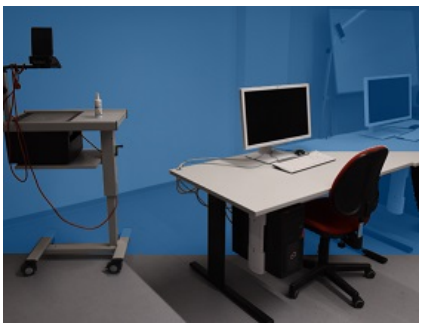

(c) Depth plane

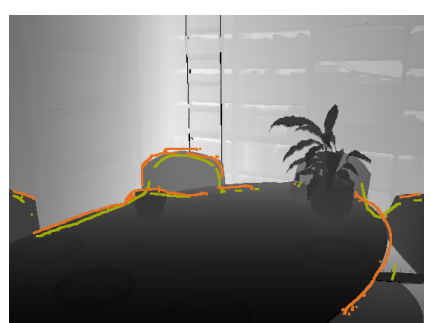

(b) Predictions

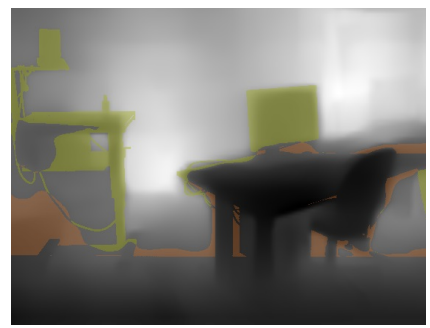

(d) Differences
Figure 7: Visual results after applying DBE $(a+b)$ and DDE $(c+d)$ on IBims -1 : (a) ground truth edge (_ $)$ for one example from the IBims-1 dataset. (b) Edge predictions using the methods of Li et al. [19] (__ ) and Laina et al. [16] (— ). (c) Ground truth depth plane at $d=3 \mathrm{~m}$ separating foreground from background ( $\square$ ). (d) Differences between ground truth and predicted depths using the method of Li et al. [19]. Color coded are depth values that are either estimated too short ( $\square$ ) or too far ( $\square$ )

\subsubsection{Directed Depth Error}

The DDE aims to identify predicted depth values which lie on the correct side of a predefined reference plane but also distinguishes between overestimated and underestimated predicted depths. This measure could be useful for applications like 3D cinematography, where a 3D effect is generated by defining two depth planes. For this experiment, we defined a reference plane at $3 \mathrm{~m}$ distance and computed the proportions of correct $\varepsilon_{\mathrm{DDE}}^{0}$, overestimated $\varepsilon_{\mathrm{DDE}}^{+}$, and underestimated $\varepsilon_{\mathrm{DDE}}^{-}$ depth values towards this plane according to the error definitions in Section 3.2.4. Table 3 lists the resulting proportions for IBims-1, while a visual illustration of correctly and falsely predicted depths is depicted in Figs. $7 \mathrm{c}$ and $7 \mathrm{~d}$. The results show that the methods tended to predict depths to a too short distance, although the number of correctly estimated depths almost reaches $90 \%$ and $80 \%$ for NYU-V2 and IBims-1, respectively. 
Table 4: Quantitative results on the augmented IBims-1 dataset exemplary listed for the global relative distance error. Errors showing relative differences for various image augmentations towards the error of the predicted original input image (Ref).

\begin{tabular}{|c|c|c|c|c|c|c|c|c|c|c|c|c|}
\hline \multirow[t]{2}{*}{ Method } & \multirow[t]{2}{*}{ Ref. } & \multicolumn{2}{|c|}{ Geometric } & \multicolumn{3}{|l|}{ Contrast } & \multicolumn{2}{|c|}{ Ch. Swap } & \multicolumn{2}{|l|}{ Hue } & \multicolumn{2}{|c|}{ Saturation } \\
\hline & & LR & UD & $\gamma=0.2$ & $\gamma=2$ & Norm. & GBR & BRG & $+9^{\circ}$ & $+90^{\circ}$ & $\times 0.9$ & $\times 0$ \\
\hline Eigen [7] & 0.360 & -0.024 & 0.068 & 0.059 & 0.019 & -0.001 & 0.020 & 0.010 & 0.010 & 0.025 & -0.001 & 0 \\
\hline Eigen (AlexNet) [6] & 0.318 & -0.012 & 0.113 & 0.111 & 0.031 & 0.041 & 0.022 & 0.008 & 0.005 & 0.022 & -0.001 & 0 \\
\hline Eigen (VGG) [6] & 0.288 & -0.017 & 0.137 & 0.110 & 0.014 & 0.000 & 0.005 & 0.011 & 0.002 & 0.006 & -0.001 & 0 \\
\hline Laina [16] & 0.274 & -0.018 & 0.162 & 0.079 & 0.027 & -0.001 & 0.005 & 0.008 & 0.001 & 0.004 & -0.001 & 0 \\
\hline
\end{tabular}
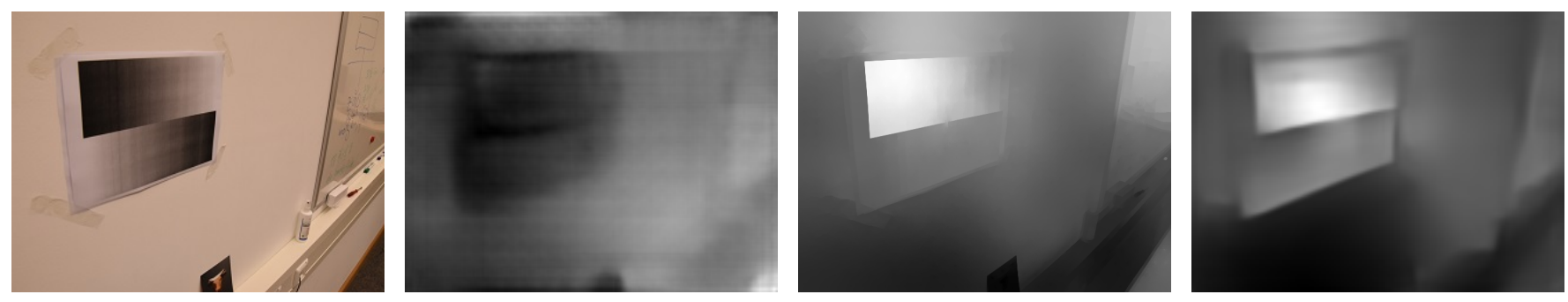

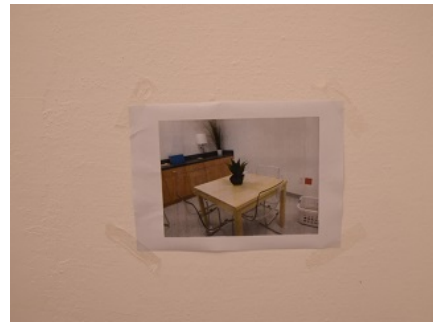

(a) RGB

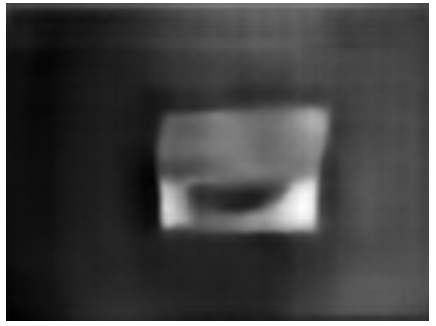

(b) Laina et al. [16]

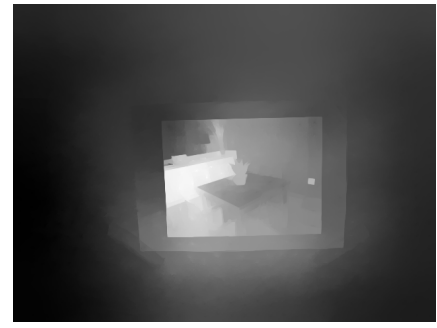

(c) Liu et al. [20]

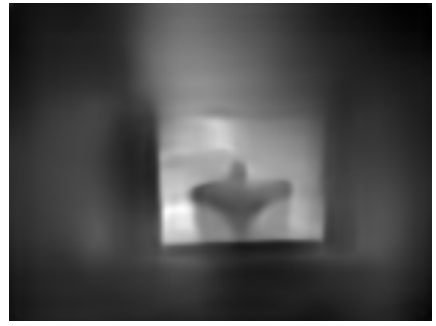

(d) Eigen [6]

Figure 8: Predicted depths for two samples from the auxiliary part of the proposed IBims-1 dataset showing printed samples from the Patterns [1] dataset (top) and the NYU-V2 dataset [28] (bottom) on a planar surface

\subsection{Further Analyses}

A series of additional experiments were conducted to investigate the behavior of SIDE methods in special situations. The challenges cover an augmentation of our dataset with various color and geometrical transformations and an auxiliary dataset containing images of printed patterns and $\mathrm{NYU}-\mathrm{v} 2$ images on a planar surface.

\subsubsection{Data Augmentation}

In order to assess the robustness of SIDE methods w.r.t. simple geometrical and color transformation and noise, we derived a set of augmented images from our dataset. For geometrical transformations we flipped the input images horizontally - which is expected to not change the results significantly-and vertically, which is expected to expose slight overfitting effects. As images in the NYU-v2 dataset usually show a considerable amount of pixels on the floor in the lower part of the picture, this is expected to notably influence the estimated depth maps. For color transformations, we consider swapping of image channels, shifting the hue by some offset $h$ and scaling the saturation by a factor $s$. We change the gamma values to simulate overand under-exposure and optimize the contrast by histogram stretching. Blurred versions of the images are simulated by applying gaussian blur with increasing standard deviation $\sigma$. Furthermore, we consider noisy versions of the images by ap- plying gaussian additive noise and salt and pepper noise with increasing variance and amount of affected pixels, respectively.

Table 4 shows results for these augmented images using the global relative error metric for selected methods. As expected, the geometrical transformations yielded contrasting results. While the horizontal flipping did not influence the results by a large margin, flipping the images vertically increased the error by up to $60 \%$. Slight overexposure influenced the result notably, underexposure seems to have been less problematic. Histogram stretching had no influence on the results, suggesting that this is already a fixed or learned part of the methods. The methods also seem to be robust to color changes, which is best seen in the results for $s=0$, i.e., , greyscale input images which yielded an equal error to the reference.

\subsubsection{Textured Planar Surfaces}

Experiments with printed patterns and NYU-v2 samples on a planar surface exploit the most important features useful for SIDE. As to be seen in the first example in Fig. A12, gradients seem to serve as a strong hint to the network. All of the tested methods estimated incorrectly depth in the depicted scene, none of them, however, identified the actual planarity of the picture. 


\section{Conclusions}

We presented a novel set of quality criteria for the evaluation of SIDE methods. Furthermore, we introduced a new high-quality dataset, fulfilling the need for an extended ground truth of our proposed metrics. Using this test protocol we evaluated and compared state-of-the-art SIDE methods. In our experiments, we were able to assess the quality of the compared approaches w.r.t. to various meaningful properties, such as the preservation of edges and planar regions, depth consistency, and absolute distance accuracy. Compared to commonly used global metrics, our proposed set of quality criteria enabled us to unveil even subtle differences between the considered SIDE methods. In particular, our experiments have shown that the prediction of planar surfaces, which is crucial for many applications, is lacking accuracy. Furthermore, edges in the predicted depth maps tend to be oversmooth for many methods. We believe that our dataset is suitable for future developments in this regard, as our images are provided in a very high resolution and contain new sceneries with extended scene depths.

The IBims-1 dataset can be downloaded at www. lmf. bgu . tum.de/ibims1.

\section{Acknowledgements}

This research was funded by the German Research Foundation (DFG) for Tobias Koch and the Federal Ministry of Transport and Digital Infrastructure (BMVI) for Lukas Liebel. We thank our colleagues from the Chair of Geodesy for providing all the necessary equipment and our student assistant Leonidas Stöckle for his help during the data acquisition campaign.

\section{References}

[1] Nicola Asuni and Andrea Giachetti. TESTIMAGES: a Large-scale Archive for Testing Visual Devices and Basic Image Processing Algorithms. In Andrea Giachetti, editor, Smart Tools and Apps for Graphics - Eurographics Italian Chapter Conference. The Eurographics Association, 2014. ISBN 978-3-905674-72-9. doi: 10.2312/stag.20141242.

[2] Heinrich H. Bülthoff and Alan L. Yuille. Shape from x: Psychophysics and computation. In Michael Landy and J. Anthony Movshon, editors, Computational Models of Visual Processing, pages 305-330. MIT Press, 1991.

[3] Ayan Chakrabarti, Jingyu Shao, and Greg Shakhnarovich. Depth from a single image by harmonizing overcomplete local network predictions. In Advances in Neural Information Processing Systems, pages 2658-2666, 2016.

[4] Angel Chang, Angela Dai, Thomas Funkhouser, Maciej Halber, Matthias Nießner, Manolis Savva, Shuran Song, Andy Zeng, and Yinda Zhang. Matterport3d: Learning from rgb-d data in indoor environments. arXiv preprint arXiv:1709.06158, 2017.

[5] Piotr Dollár and C Lawrence Zitnick. Fast edge detection using structured forests. IEEE transactions on pat- tern analysis and machine intelligence, 37(8):1558-1570, 2015.

[6] David Eigen and Rob Fergus. Predicting depth, surface normals and semantic labels with a common multi-scale convolutional architecture. In Proceedings of the IEEE International Conference on Computer Vision, pages 2650 2658, 2015. doi: 10.1109/ICCV.2015.304.

[7] David Eigen, Christian Puhrsch, and Rob Fergus. Depth map prediction from a single image using a multi-scale deep network. In International Conference on Neural Information Processing Systems, volume 2, pages 23662374, 2014.

[8] Paolo Favaro and Stefano Soatto. A geometric approach to shape from defocus. IEEE Transactions on Pattern Analysis and Machine Intelligence, 27(3):406-417, 2005. doi: 10.1109/TPAMI.2005.43.

[9] Ravi Garg, Gustavo Carneiro, and Ian Reid. Unsupervised cnn for single view depth estimation: Geometry to the rescue. In European Conference on Computer Vision, pages 740-756. Springer, 2016. doi: 10.1007/ 978-3-319-46484-8_45.

[10] Andreas Geiger, Philip Lenz, and Raquel Urtasun. Are we ready for autonomous driving? the kitti vision benchmark suite. In Computer Vision and Pattern Recognition (CVPR), 2012 IEEE Conference on, pages 3354-3361. IEEE, 2012.

[11] Stefan Heber and Thomas Pock. Convolutional networks for shape from light field. In IEEE Conference on Computer Vision and Pattern Recognition, pages 3746-3754, June 2016. doi: 10.1109/CVPR.2016.407.

[12] Berthold K. P. Horn. Shape from shading: A method for obtaining the shape of a smooth opaque object from one view. Technical report, MIT, Cambridge, MA, USA, 1970. URL https://dspace.mit.edu/handle/1721. $1 / 6885$.

[13] Achuta Kadambi, Vage Taamazyan, Boxin Shi, and Ramesh Raskar. Polarized 3d: High-quality depth sensing with polarization cues. In IEEE International Conference on Computer Vision, pages 3370-3378, 2015. doi: 10.1109/ICCV.2015.385.

[14] Seungryong Kim, Kihong Park, Kwanghoon Sohn, and Stephen Lin. Unified depth prediction and intrinsic image decomposition from a single image via joint convolutional neural fields. In European Conference on Computer Vision, pages 143-159. Springer, 2016.

[15] Arno Knapitsch, Jaesik Park, Qian-Yi Zhou, and Vladlen Koltun. Tanks and temples: Benchmarking large-scale scene reconstruction. ACM Transactions on Graphics, 36 (4), 2017. 
[16] Iro Laina, Christian Rupprecht, Vasileios Belagiannis, Federico Tombari, and Nassir Navab. Deeper depth prediction with fully convolutional residual networks. CoRR, abs/1606.00373, 2016. doi: 10.1109/3dv.2016.32. URL http: / / arxiv.org/abs/1606.00373.

[17] Bo Li, Chunhua Shen, Yuchao Dai, Anton van den Hengel, and Mingyi He. Depth and surface normal estimation from monocular images using regression on deep features and hierarchical crfs. In Proceedings of the IEEE Conference on Computer Vision and Pattern Recognition, pages 1119 $1127,2015$.

[18] Bo Li, Yuchao Dai, Huahui Chen, and Mingyi He. Single image depth estimation by dilated deep residual convolutional neural network and soft-weight-sum inference. arXiv preprint arXiv:1705.00534, 2017.

[19] Jun Li, Reinhard Klein, and Angela Yao. A two-streamed network for estimating fine-scaled depth maps from single rgb images. In Proceedings of the IEEE Conference on Computer Vision and Pattern Recognition, pages 33723380, 2017.

[20] Fayao Liu, Chunhua Shen, and Guosheng Lin. Deep convolutional neural fields for depth estimation from a single image. In Proceedings of the IEEE Conference on Computer Vision and Pattern Recognition, pages 51625170, 2015.

[21] Fayao Liu, Chunhua Shen, Guosheng Lin, and Ian Reid. Learning depth from single monocular images using deep convolutional neural fields. IEEE transactions on pattern analysis and machine intelligence, 38(10):2024-2039, 2016.

[22] Francesc Moreno-Noguer, Vincent Lepetit, and Pascal Fua. Accurate non-iterative o (n) solution to the pnp problem. In Computer vision, 2007. ICCV 2007. IEEE 11th international conference on, pages 1-8. IEEE, 2007.

[23] Trung Thanh Ngo, Hajime Nagahara, and Rin-ichiro Taniguchi. Shape and light directions from shading and polarization. In IEEE Conference on Computer Vision and Pattern Recognition, pages 2310-2318, 2015. doi: 10.1109/CVPR.2015.7298844.

[24] Anirban Roy and Sinisa Todorovic. Monocular depth estimation using neural regression forest. In Proceedings of the IEEE Conference on Computer Vision and Pattern Recognition, pages 5506-5514, 2016. doi: 10.1109/cvpr. 2016.594.

[25] Ashutosh Saxena, Min Sun, and Andrew Y Ng. Make3d: Learning $3 \mathrm{~d}$ scene structure from a single still image. IEEE transactions on pattern analysis and machine intelligence, 31(5):824-840, 2009.

[26] Thomas Schöps, Johannes L Schönberger, Silvano Galliani, Torsten Sattler, Konrad Schindler, Marc Pollefeys, and Andreas Geiger. A multi-view stereo benchmark with high-resolution images and multi-camera videos. In Proc. CVPR, 2017.

[27] Steven M. Seitz, Brian Curless, James Diebel, Daniel Scharstein, and Richard Szeliski. A comparison and evaluation of multi-view stereo reconstruction algorithms. In Computer Society Conference on Computer Vision and Pattern Recognition, volume 1, pages 519-528, 2006. doi: 10.1109/CVPR.2006.19.

[28] Nathan Silberman, Derek Hoiem, Pushmeet Kohli, and Rob Fergus. Indoor segmentation and support inference from rgbd images. Computer Vision-ECCV 2012, pages 746-760, 2012.

[29] Christoph Strecha, Wolfgang Von Hansen, Luc Van Gool, Pascal Fua, and Ulrich Thoennessen. On benchmarking camera calibration and multi-view stereo for high resolution imagery. In Computer Vision and Pattern Recognition, 2008. CVPR 2008. IEEE Conference on, pages 1-8. Ieee, 2008.

[30] Supasorn Suwajanakorn, Carlos Hernandez, and Steven M. Seitz. Depth from focus with your mobile phone. In IEEE Conference on Computer Vision and Pattern Recognition, pages 3497-3506, 2015. doi: 10.1109/CVPR.2015. 7298972 .

[31] Keisuke Tateno, Federico Tombari, Iro Laina, and Nassir Navab. Cnn-slam: Real-time dense monocular slam with learned depth prediction. arXiv preprint arXiv:1704.03489, 2017.

[32] Andrea J. van Doorn, Jan J. Koenderink, and Johan Wagemans. Light fields and shape from shading. Journal of Vision, 11(3):21.1-21.21, 2011. doi: 10.1167/11.3.21.

[33] Peng Wang, Xiaohui Shen, Zhe Lin, Scott Cohen, Brian Price, and Alan L Yuille. Towards unified depth and semantic prediction from a single image. In Proceedings of the IEEE Conference on Computer Vision and Pattern Recognition, pages 2800-2809, 2015.

[34] Dan Xu, Elisa Ricci, Wanli Ouyang, Xiaogang Wang, and Nicu Sebe. Multi-scale continuous crfs as sequential deep networks for monocular depth estimation. arXiv preprint arXiv:1704.02157, 2017

[35] Ruo Zhang, Ping-Sing Tsai, James Edwin Cryer, and Mubarak Shah. Shape from shading: A survey. IEEE Transactions on Pattern Analysis and Machine Intelligence, 21(8):690-706, 1999. doi: 10.1109/34.784284. 


\title{
Supplementary Material for Evaluation of CNN-based Single-Image Depth Estimation Methods
}

\author{
Tobias Koch $^{1} \quad$ Lukas Liebel $^{1} \quad$ Friedrich Fraundorfer $^{23} \quad$ Marco Körner $^{1}$ \\ ${ }^{1}$ Chair of Remote Sensing Technology, Computer Vision Research Group, Technical University of Munich \\ \{tobias.koch, lukas.liebel, marco.koerner\} @ tum.de \\ ${ }^{2}$ Institute for Computer Graphics and Vision, Graz University of Technology \\ fraundorfer@icg.tugraz.at \\ ${ }^{3}$ Remote Sensing Technology Institute (IMF), German Aerospace Center (DLR)
}

In this supplementary document, we provide further information on different sections of the paper and present additional visualizations (i.a. sample images of our IBims-1 dataset and provided masks, our data acquisition setup, and qualitative results of the evaluation part). The numbering and naming of following sections refer to the section naming in the paper.

\section{Dataset}

The following sections comprise additional descriptions of our dataset and present several sample images and annotations of IBims-1.

\subsection{Acquisition}

For the creation of depth maps, we considered various sensors and instruments. Common mass market RGB-D products, such as Microsoft Kinect, not only allow for fast and convenient capturing of scenes, but also provide registered images and depth maps at the same time. However, the overall qualityespecially in terms of resolution and accuracy-of the resulting depth maps and images turn out to be insufficient for the intended usage as reference data. Due to the low maximum range and high sensitivity to sunlight, the Kinect is only suitable for indoor applications. Stereo rigs, such as the Stereolabs ZED camera, outperform RGB-D products in several crucial areas. They are equally easy to use but also show deficits in certain areas. As the stereo reconstruction only produces results for textured surfaces, the produced depth maps are often incomplete and suffer from noise. Precise geodetic instruments, such as tacheometers, laser trackers, or laser scanners, can provide highly accurate distance measurements. Laser scanners excel in recording highly accurate dense point clouds. Fig. A1 shows a comparison of depth maps acquired from different sensors As we want to generate highly accurate depth maps for highresolution images, we chose a laser scanner as our sensor of choice. They do, however, fall short of expectations regarding provided imagery. As only a few instruments can capture RGB

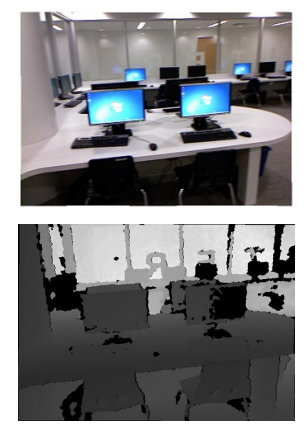

(a) Kinect v1
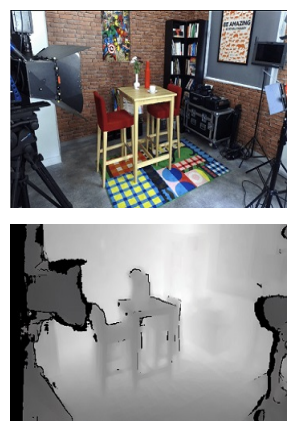

(b) ZED Cam

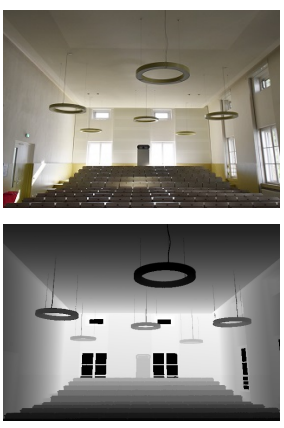

(c) Laser scanner

Figure A1: Sample RGB images (top) and corresponding depth maps (bottom) taken with different sensors

images at all, this is, in practice, most commonly done using an auxiliary camera. For this reason, we decided to design our acquisition setup as it is already explained in the paper. An illustration of our setup is depicted in Fig. A2.

\subsection{Contents}

In addition to already shown sample images of our dataset in the paper, further examples can be taken from Fig. A3.

All images are provided with different masks which are further used in the evaluation procedures. Unreliable or invalid pixels in the depth map are labeled by two different sets of binary masks. One of which flags transparent objects, mainly windows, which could be assigned with an ambiguous depth. While the laser scanner captured points behind those objects, it may be intended to obtain the distance of the transparent object for certain applications. The other mask for invalid pixels indicates faulty values in the 3D point cloud. Those mainly originate from scanner-related errors, such as reflecting surfaces, as well as regions out of range. Three further sets of masks label planar surfaces of three different types, i.e., tables, floors, and walls (cf. Fig. A4 for examples). Each instance is contained in a 


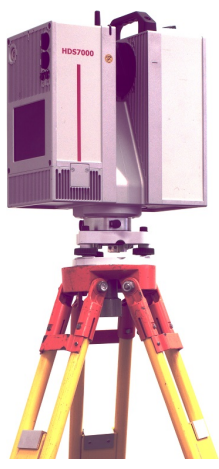

(a) Laser scanner

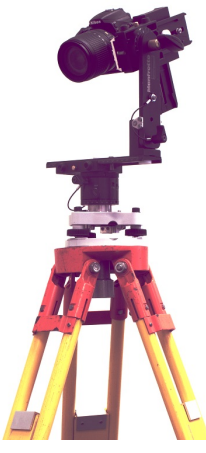

(b) Camera
Figure A2: Our hardware setup used for the acquisition of IBims-1 with a laser scanner (a) and a DSLR camera (b) mounted on a survey tripod. A custom panoramic tripod is used in order to achieve a coincidence of the optical center of the camera and the origin of the laser scanner coordinate system to avoid gaps in the resulting depth maps

separate mask.

Examples of provided edge masks are shown in Fig. A5.

\section{Evaluation}

The following sections contain additional quantitative and qualitative results of our evaluation.

\subsubsection{Distance-Related Assessment}

In addition to the distance-related evaluation, Table 5 lists the global scores on the NYU-V2 and IBims-1 dataset by computing the statistical error metrics on the complete images. This is the standard evaluation procedure in all recent publications. The revealed lower overall scores for our dataset are expected since the dataset is previously unseen by these methods. As the methods are trained to predict depths in the range of the NYU-V2 dataset (i.e., 1-10 m), they are not able to estimate depths beyond this range which are also encompassed in our dataset. The average RMS error increases to about $2.5 \mathrm{~m}$. The method proposed by $\mathrm{Li}$ et al. [19] achieved best results on IBims-1 and ranked second on the NYU-v2 dataset.

\subsubsection{Planarity}

A more detailed analysis of the performance of selected SIDE methods according to our proposed planarity metrics is compiled in Table 6 . In addition to the combined errors comprising all masks, as listed in the paper, planarity errors are given for horizontal and vertical planes separately. Visual illustrations of the resulting 3D point clouds and fitted 3D planes for two sample images are depicted in Figs. A6 and A7.

\subsubsection{Data Augmentation}

As a completion of the analysis of the augmented dataset, Fig. A8 shows the results after blurring and adding noise to the images, while samples of augmented images and their depth predictions are given in Figs. A9 to A11. Fig. A8a shows a no- table peak when blurring with a moderate-sized kernel. Minor blurring did not change the results, as the examined methods considerably down-sample the input images and are thus robust to blurring up to a certain kernel size. While the increasing error for bigger kernel sizes was expected, the distinct peak could hint at discretization effects. The results for adding noise to the images, shown in Figs. A8b and A8c, give certain thresholds for the maximum tolerable amount of noise. All of the considered methods were able to cope with up to $10 \%$ of noise until the quality of results decreased notably. The tested methods responded to the augmentations accordingly. The AlexNet version of Eigen and Fergus [6] seems to be more robust to noise as opposed to the VGG version, which is, however, less sensitive to blurred input images.

\subsubsection{Textured Planar Surfaces}

Further qualitatively results of the printed samples from the Pattern dataset [1] are shown in Fig. A12. All of the examined networks respond to these patterns. However, this effect is less severe for Laina et al. [16], which respond with only a constant offset to the alternating gradients in the pattern. Edges in the input also seem to influence the result as to be seen in Stripes and Boxes Again, Laina et al. [16] gave a constant offset, while the result of [21] clearly contained artifacts of the superpixel approach, which is even more evident in Curves. A clearer visualization is obtained by projecting the depth maps to $3 \mathrm{D}$ point clouds, which are depicted in Fig. A13. 

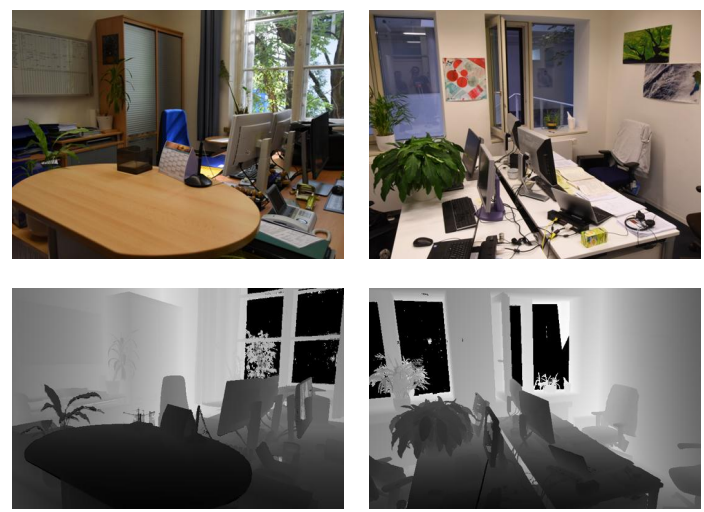

(a) Office Room 1
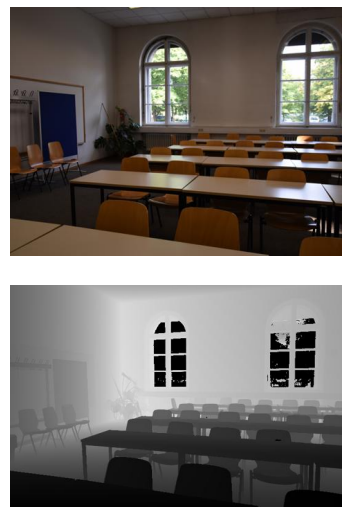

(f) Classroom 1
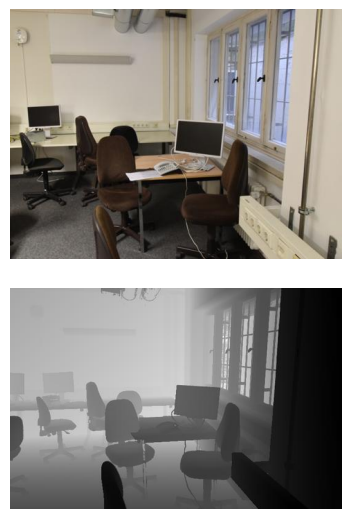

(k) Computer Lab 1
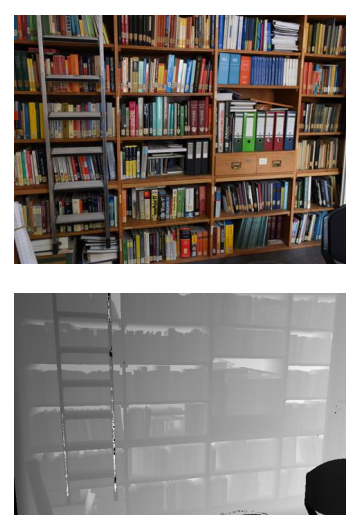

(p) Library 1

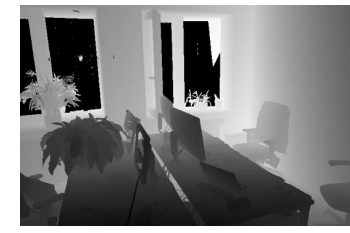

(b) Office Room 2
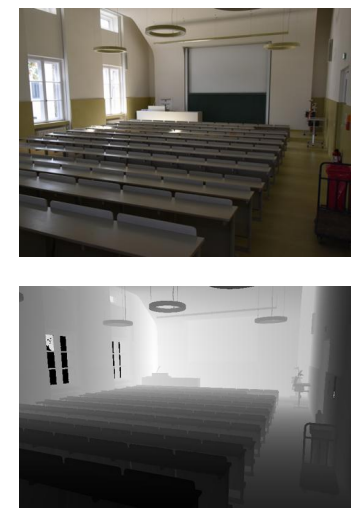

(g) Classroom 2
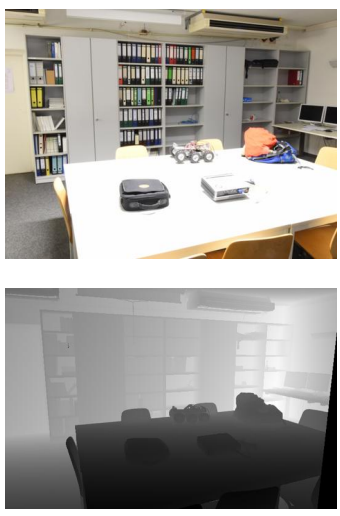

(l) Computer Lab 2
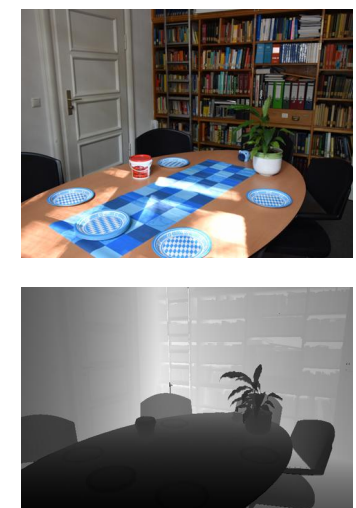

(q) Library 2
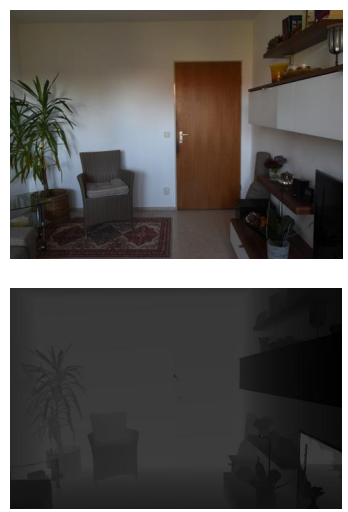

(c) Livingroom 1
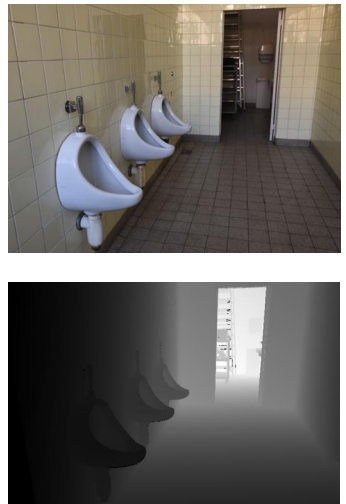

(h) Restroom 1
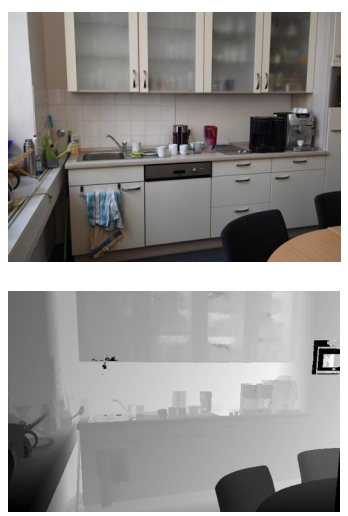

(m) Kitchen 1
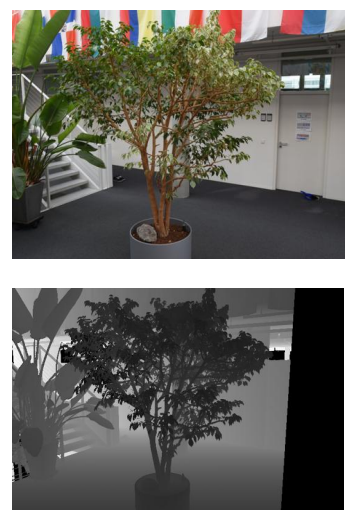

(r) Various 4
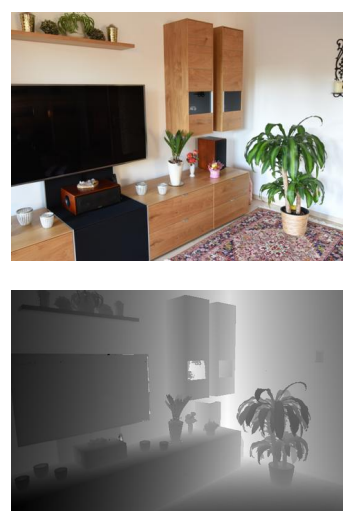

(d) Livingroom 2
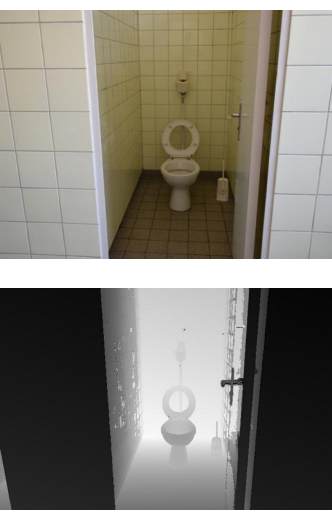

(i) Restroom 2
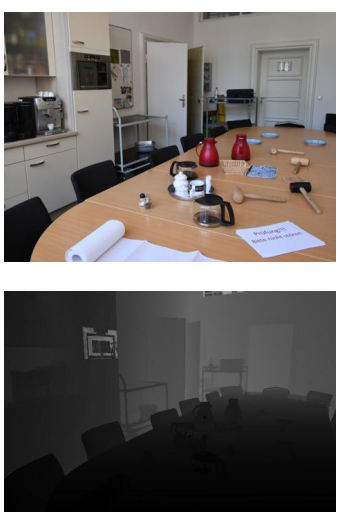

(n) Kitchen 2
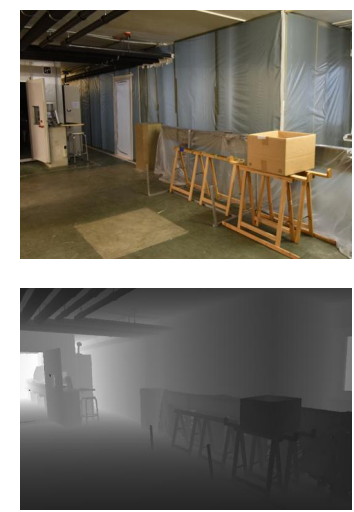

(s) Various 5
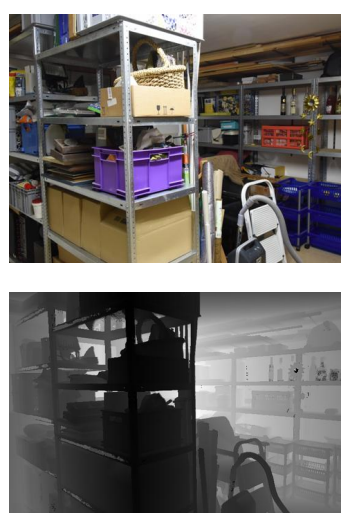

(e) Various 1
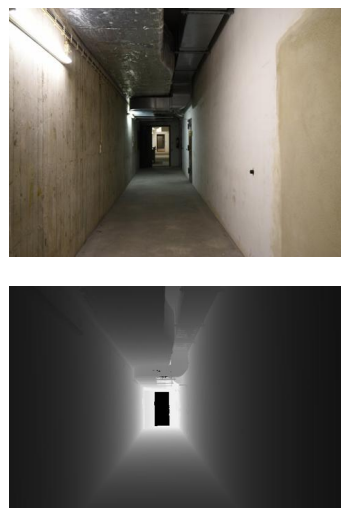

(j) Various 2
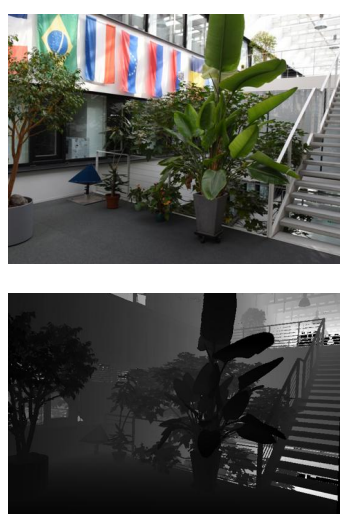

(o) Various 3
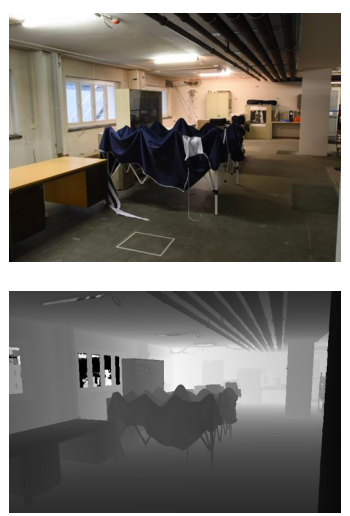

(t) Various 6

Figure A3: Examples from the proposed IBims-1 dataset containing different scenarios with RGB images (top) and ground truth depth maps (bottom) 

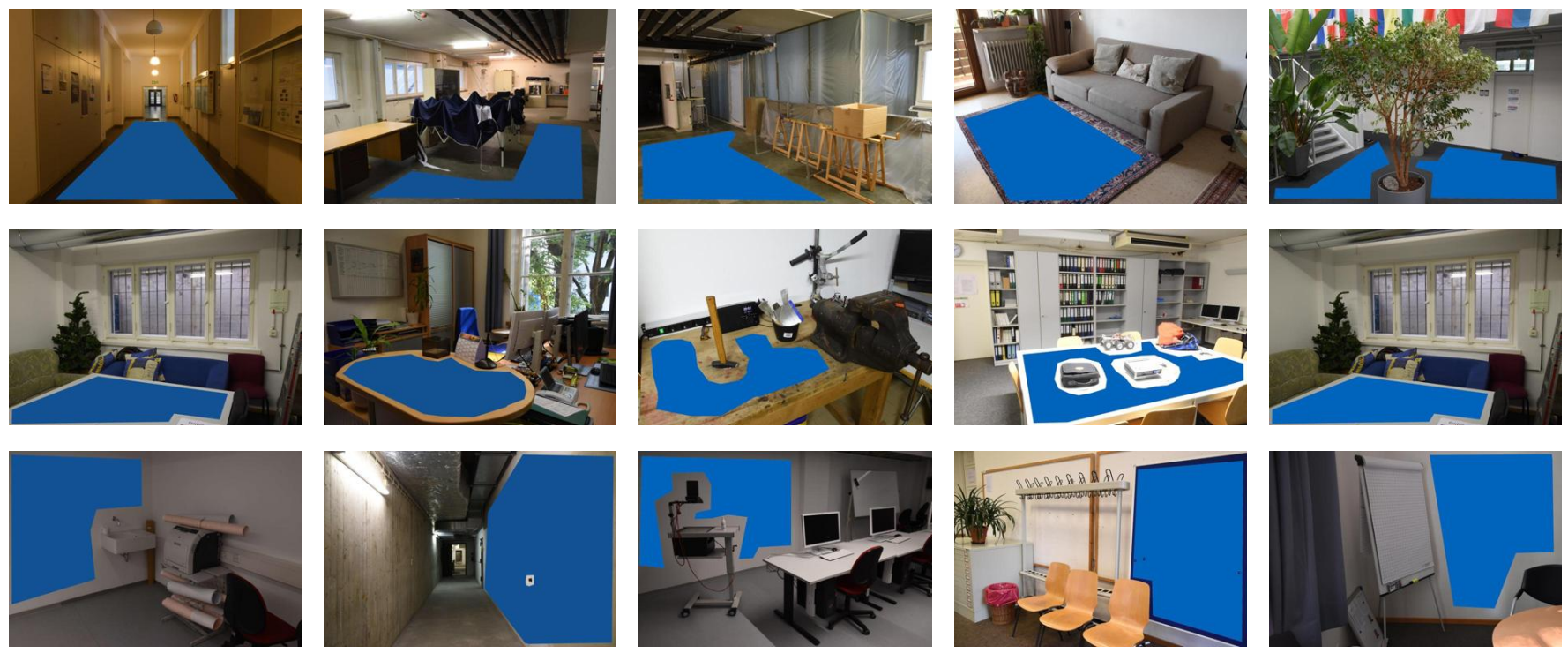

Figure A4: Annotation samples showing provided plane masks for floors (top), tables (mid) and walls (bottom)
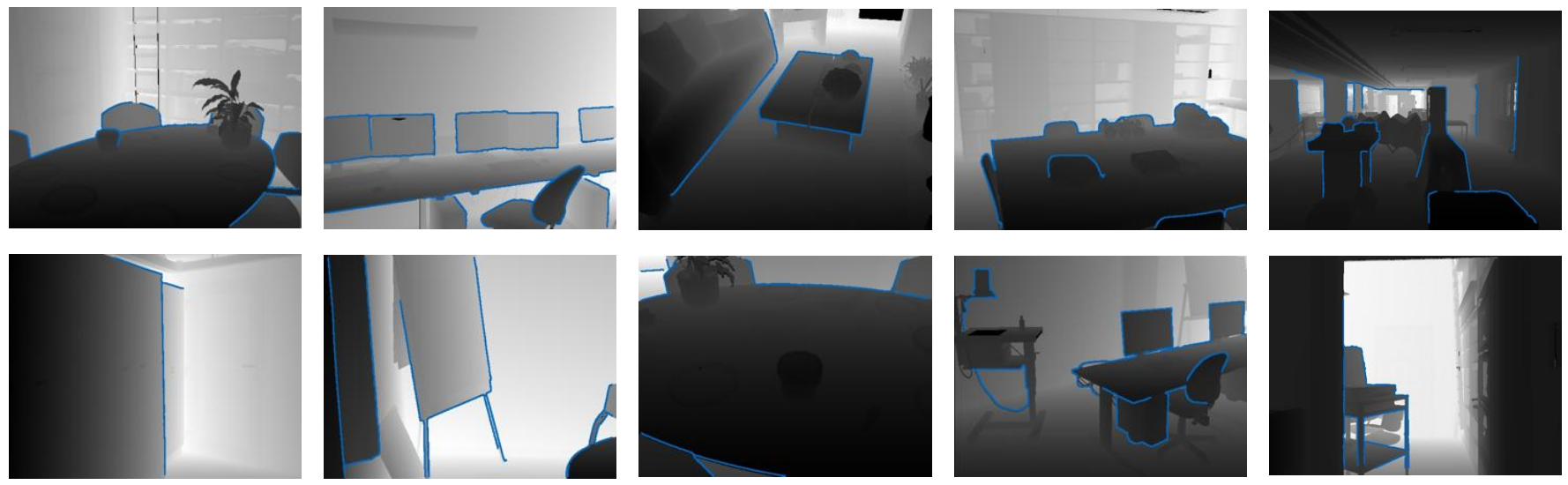

Figure A5: Annotation samples showing provided edge masks (blue)

Table 5: Results for the NYU-v2 and IBims-1 datasets using standard global error metrics (lower values for rel, $\log _{10}$ and RMS indicate better results; larger values for the threshold metrics indicate better results)

\begin{tabular}{|c|c|c|c|c|c|c|c|}
\hline \multirow[t]{2}{*}{ Method } & \multirow[t]{2}{*}{ Dataset } & \multirow[t]{2}{*}{ rel } & \multirow[t]{2}{*}{$\log _{10}$} & \multirow[t]{2}{*}{ RMS } & \multicolumn{3}{|c|}{ Threshold $\left(\delta_{i}=1.25^{i}\right)$} \\
\hline & & & & & $\delta_{1}$ & $\delta_{2}$ & $\delta_{3}$ \\
\hline Eigen et al. [7] & NYU-V2 & 0.22 & 0.09 & 0.76 & 0.61 & 0.89 & 0.97 \\
\hline Eigen and Fergus [6] (AlexNet) & NYU-V2 & 0.19 & 0.08 & 0.67 & 0.69 & 0.91 & 0.98 \\
\hline Eigen and Fergus [6] (VGG) & NYU-V2 & 0.16 & 0.07 & 0.58 & 0.75 & 0.95 & 0.99 \\
\hline Laina et al. [16] & NYU-v2 & 0.14 & 0.06 & 0.51 & 0.82 & 0.95 & 0.99 \\
\hline Liu et al. [21] & NYU-v2 & 0.21 & 0.09 & 0.68 & 0.66 & 0.91 & 0.98 \\
\hline Li et al. [19] & NYU-V2 & 0.15 & 0.06 & 0.53 & 0.79 & 0.96 & 0.99 \\
\hline Eigen et al. [7] & IBims -1 & 0.36 & 0.22 & 2.92 & 0.35 & 0.63 & 0.79 \\
\hline Eigen and Fergus [6] (AlexNet) & IBims -1 & 0.32 & 0.18 & 2.63 & 0.42 & 0.72 & 0.82 \\
\hline Eigen and Fergus [6] (VGG) & IBims-1 & 0.29 & 0.17 & 2.59 & 0.47 & 0.73 & 0.85 \\
\hline Laina et al. [16] & IBims-1 & 0.27 & 0.16 & 2.42 & 0.56 & 0.76 & 0.84 \\
\hline Liu et al. [21] & IBims -1 & 0.33 & 0.17 & 2.51 & 0.46 & 0.73 & 0.84 \\
\hline Li et al. [19] & IBims -1 & 0.25 & 0.14 & 2.32 & 0.58 & 0.79 & 0.86 \\
\hline
\end{tabular}


Table 6: Results of our proposed planarity metrics for different plane types, applied to all examined methods on our proposed IBims -1 dataset

\begin{tabular}{|c|c|c|c|c|c|c|c|c|}
\hline \multirow[t]{2}{*}{ Method } & \multicolumn{2}{|c|}{ Combined } & \multicolumn{2}{|l|}{ Tables } & \multicolumn{2}{|l|}{ Floor } & \multicolumn{2}{|l|}{ Walls } \\
\hline & $\varepsilon_{\mathrm{PE}}^{\text {plan }}$ & $\varepsilon_{\mathrm{PE}}^{\text {orie }}$ & $\varepsilon_{\mathrm{PE}}^{\text {plan }}$ & $\varepsilon_{\mathrm{PE}}^{\text {orie }}$ & $\varepsilon_{\mathrm{PE}}^{\text {plan }}$ & $\varepsilon_{\mathrm{PE}}^{\text {orie }}$ & $\varepsilon_{\mathrm{PE}}^{\text {plan }}$ & $\varepsilon_{\mathrm{PE}}^{\text {orie }}$ \\
\hline Eigen et al. [7] & 0.18 & 33.27 & 0.058 & 17.96 & 0.135 & 29.29 & 0.22 & 38.38 \\
\hline Eigen and Fergus [6] (AlexNet) & 0.21 & 26.64 & 0.039 & 15.72 & 0.179 & 22.61 & 0.26 & 30.54 \\
\hline Eigen and Fergus [6] (VGG) & 0.17 & 21.64 & 0.043 & 19.92 & 0.071 & 13.48 & 0.23 & 23.88 \\
\hline Laina et al. [16] & 0.22 & 32.02 & 0.052 & 21.23 & 0.099 & 24.11 & 0.29 & 36.72 \\
\hline Liu et al. [21] & 0.22 & 31.91 & 0.049 & 21.48 & 0.098 & 22.59 & 0.30 & 36.81 \\
\hline Li et al. [19] & 0.21 & 26.67 & 0.037 & 13.16 & 0.065 & 7.77 & 0.29 & 34.60 \\
\hline
\end{tabular}

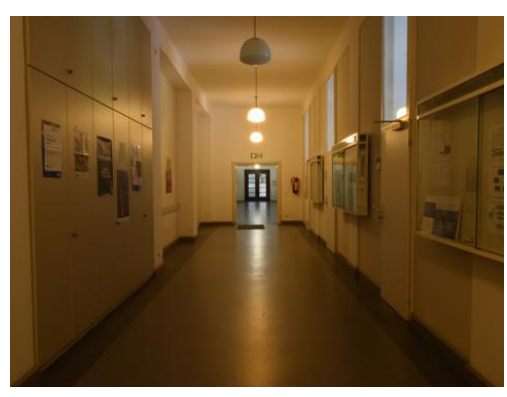

(a) RGB Image
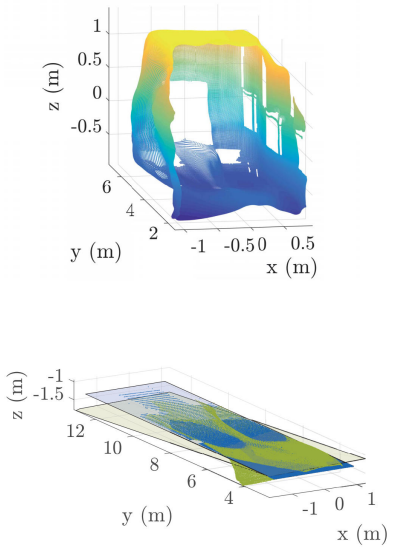

(d) Eigen and Fergus [6] (VGG):

$\varepsilon_{\mathrm{PE}}^{\text {plan }}=0.16 \mathrm{~m}$

$\varepsilon_{\mathrm{PE}}^{\text {orie }}=3.74^{\circ}$

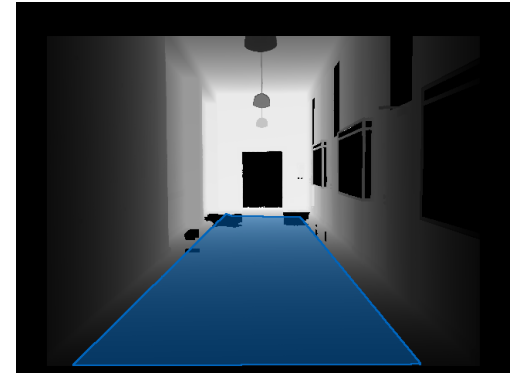

(b) Depth map with selected plane $\square$
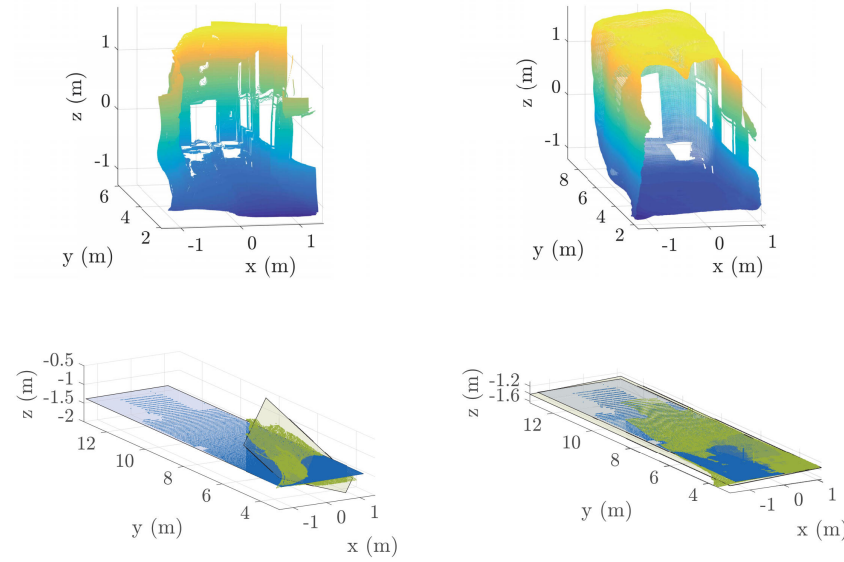

(e) Liu et al. [20]:

$\varepsilon_{\mathrm{PE}}^{\text {plan }}=0.07 \mathrm{~m}$,

$\varepsilon_{\mathrm{PE}}^{\text {orie }}=22.84^{\circ}$

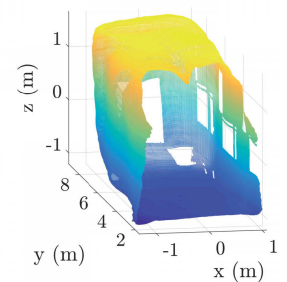

(f) Laina et al. [16]:

$\varepsilon_{\mathrm{PE}}^{\text {plan }}=0.05 \mathrm{~m}$,

$\varepsilon_{\mathrm{PE}}^{\text {orie }}=2.05^{\circ}$

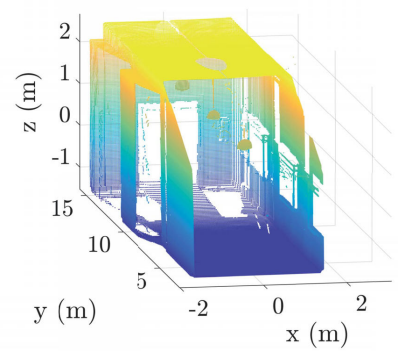

(c) Ground truth point cloud
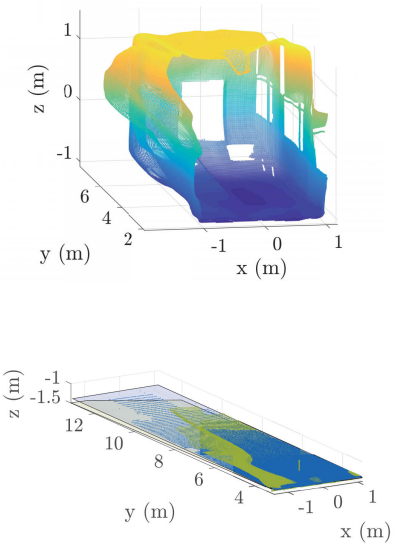

(g) Li et al. [19]:

$\varepsilon_{\mathrm{PE}}^{\text {plan }}=0.05 \mathrm{~m}$,

$\varepsilon_{\mathrm{PE}}^{\text {orie }}=2.63^{\circ}$

Figure A6: Example of our proposed planarity metric for one of the samples from the IBims-1 dataset analyzing a floor label. First row: sample RGB and depth image with floor mask and ground truth point cloud. Second row: predicted point clouds for different methods. Third row: planarity errors and fitted planes for ground truth $\square$ and predicted point clouds $\square$ 


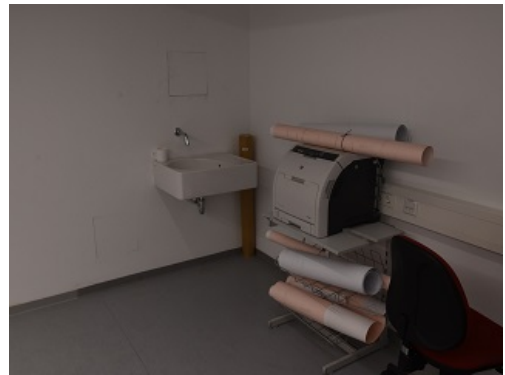

(a) RGB Image

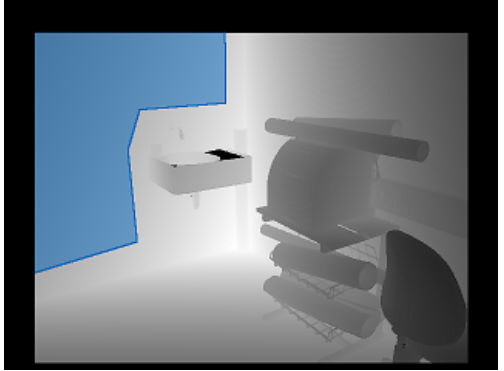

(b) Depth map with selected plane $\square$

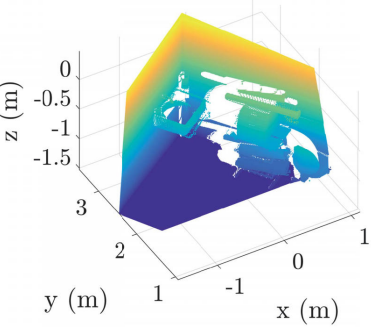

(c) Ground truth point cloud

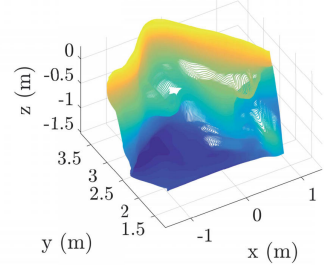

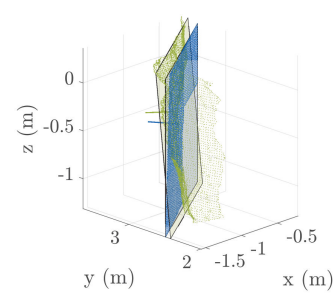

(f) Laina et al. [16]:

(e) Liu et al. [20]:

$\varepsilon_{\mathrm{PE}}^{\text {plan }}=0.08 \mathrm{~m}$, $\varepsilon_{\mathrm{PE}}^{\mathrm{Orie}}=15.02^{\circ}$

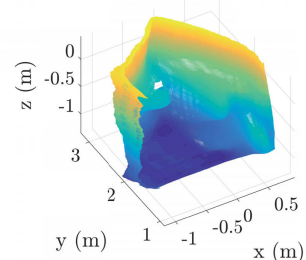

(d) Eigen and Fergus [6] (VGG): $\varepsilon_{\mathrm{PE}}^{\text {plan }}=0.09 \mathrm{~m}$, $\varepsilon_{\mathrm{PE}}^{\text {orie }}=25.60^{\circ}$
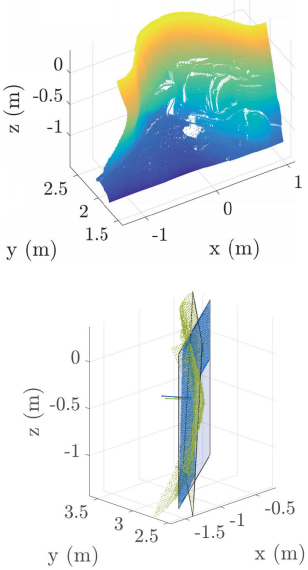

$\mathrm{x}(\mathrm{m})$

$\varepsilon_{\mathrm{PE}}^{\text {plan }}=0.15 \mathrm{~m}$,

$\varepsilon_{\mathrm{PE}}^{\text {orie }}=5.77^{\circ}$

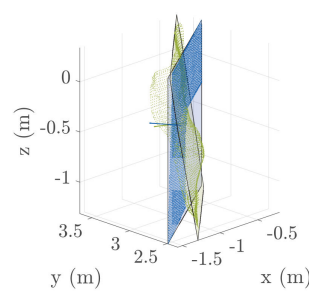

(g) Li et al. [19]:

$\varepsilon_{\mathrm{PE}}^{\text {plan }} 0.08 \mathrm{~m}$,

$\varepsilon_{\mathrm{PE}}^{\text {orie }}=13.14^{\circ}$

Figure A7: Example of our proposed planarity metric for one of the samples from the IBims-1 dataset analyzing a wall label. First row: sample RGB and depth image with wall mask and ground truth point cloud. Second row: predicted point clouds for different methods. Third row: planarity errors and fitted planes for ground truth $\square$ and predicted point clouds $\square$

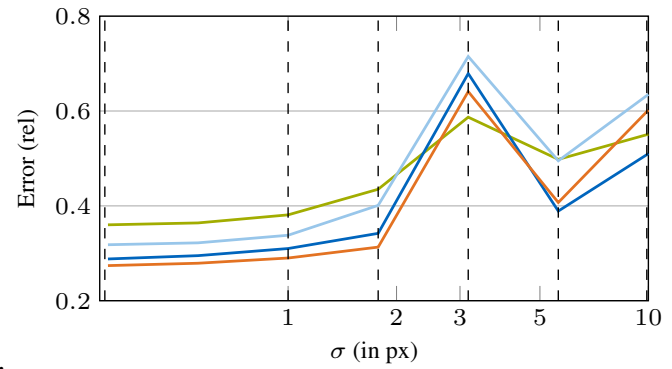

$\ddot{i} » \dot{i}$

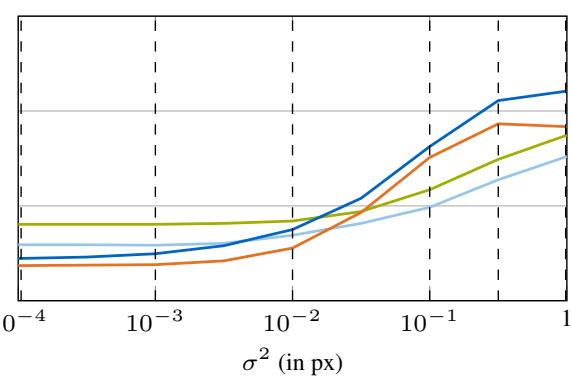

(b) Gaussian Noise

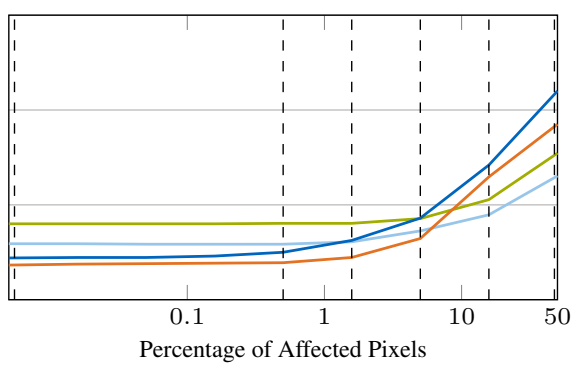

(c) Salt \& Pepper Noise

Figure A8: Quality of SIDE results, achieved using the methods proposed by Eigen et al. [7] (__ ), Eigen and Fergus [6] (AlexNet __, VGG __ ), and Laina et al. [16] (__ ) for augmentations with increasing intensity. Vertical lines (- -) correspond to the augmentation intensities of the visualizations in Figs. A9 to A11 

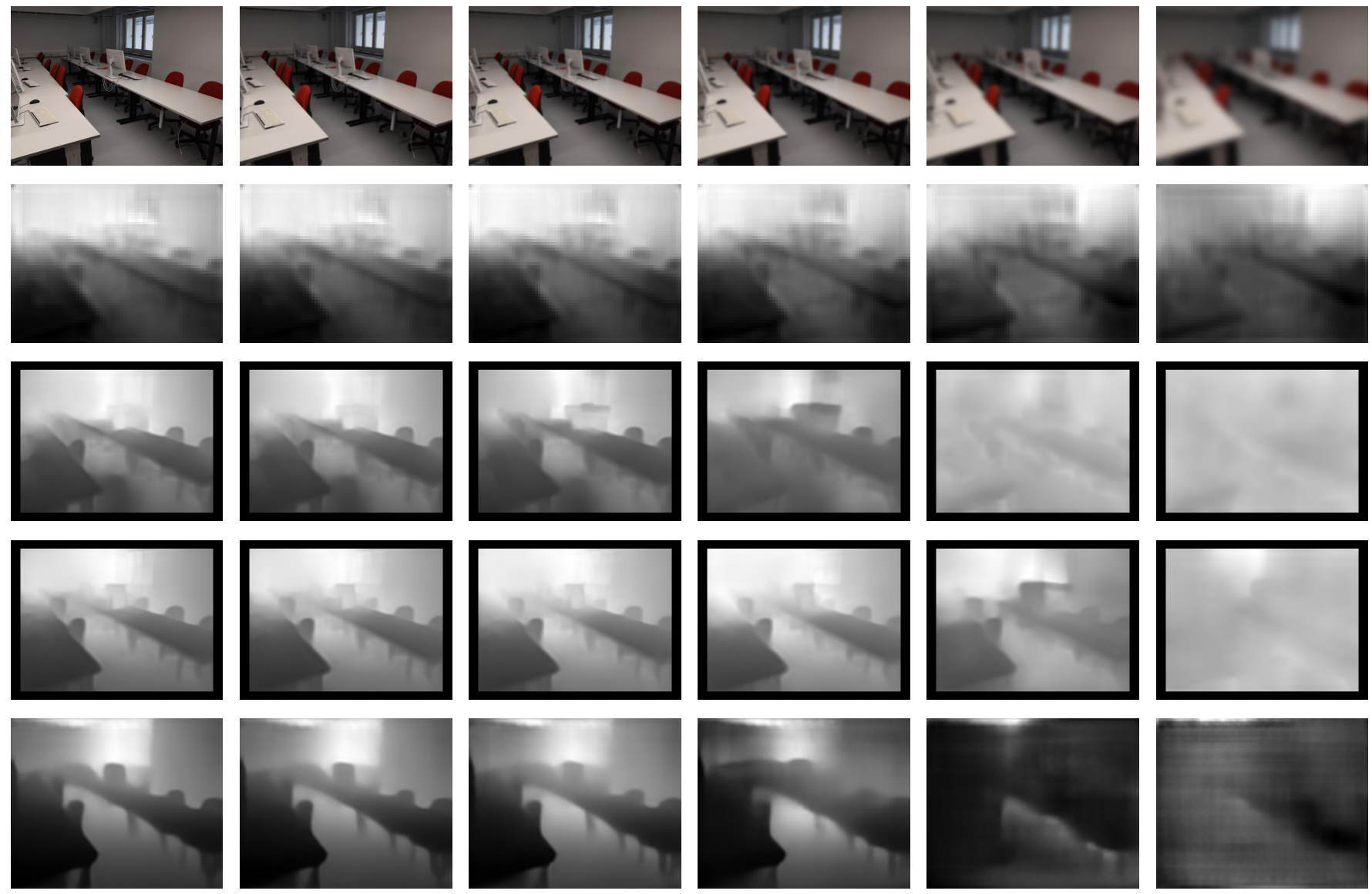

Figure A9: Depth predictions using different SIDE methods on augmented images after adding gaussian blur on the original image. Increasing blur level from left to right $(0.1,1.0,1.7783,3.1623,5.6234,10.0 \mathrm{px})$. From top to bottom: augmented RGB image, Eigen et al. [7], Eigen and Fergus [6] (AlexNet), Eigen and Fergus [6] (VGG), Laina et al. [16] 

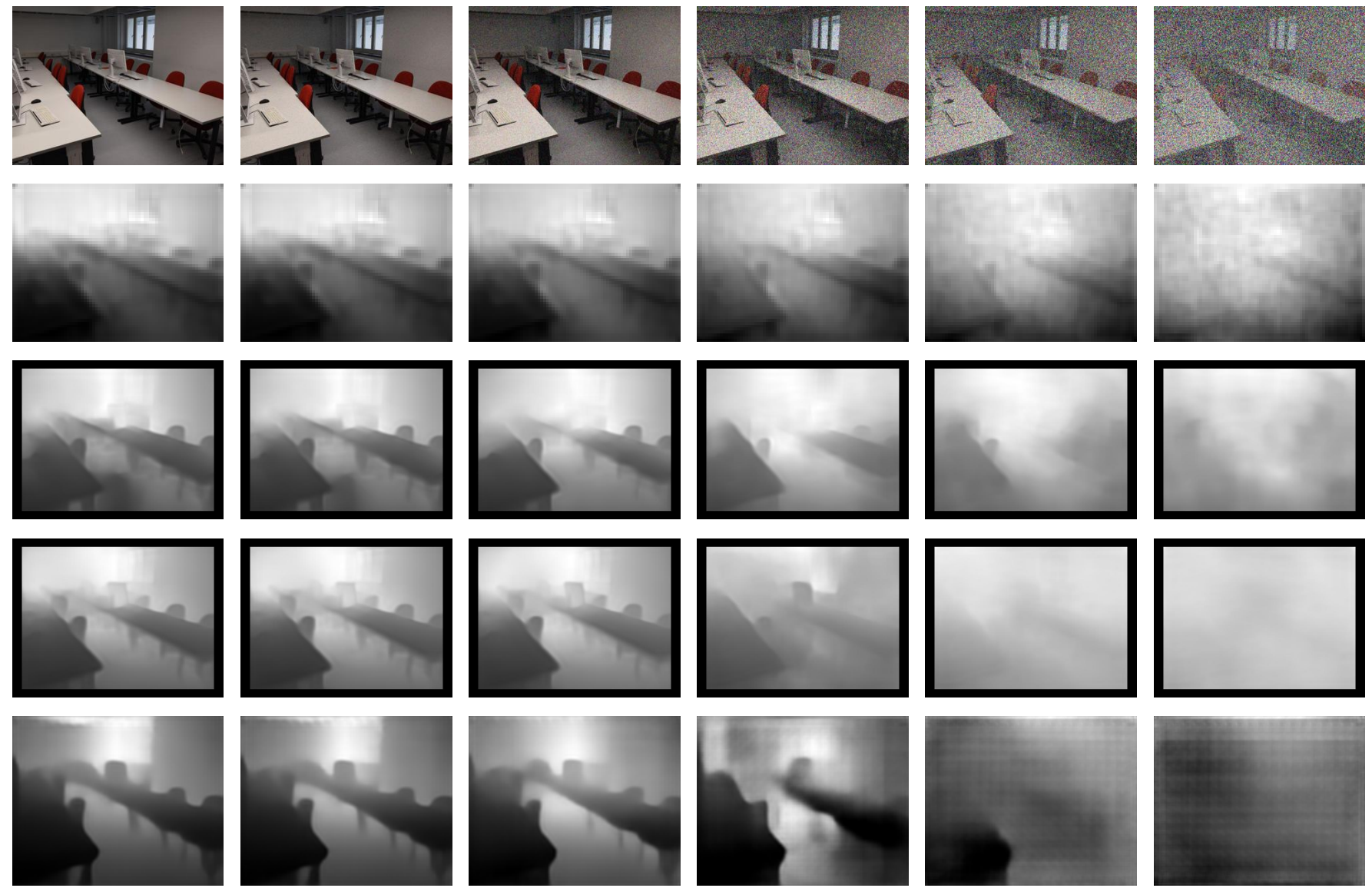

Figure A10: Depth predictions using different SIDE methods on augmented images after adding gaussian noise on the original image. Increasing noise level from left to right $\left(10^{-5}, 10^{-3}, 10^{-2}, 10^{-1}, 0.31623,1 \mathrm{px}\right)$. From top to bottom: augmented RGB image, Eigen et al. [7], Eigen and Fergus [6] (AlexNet), Eigen and Fergus [6] (VGG), Laina et al. [16] 

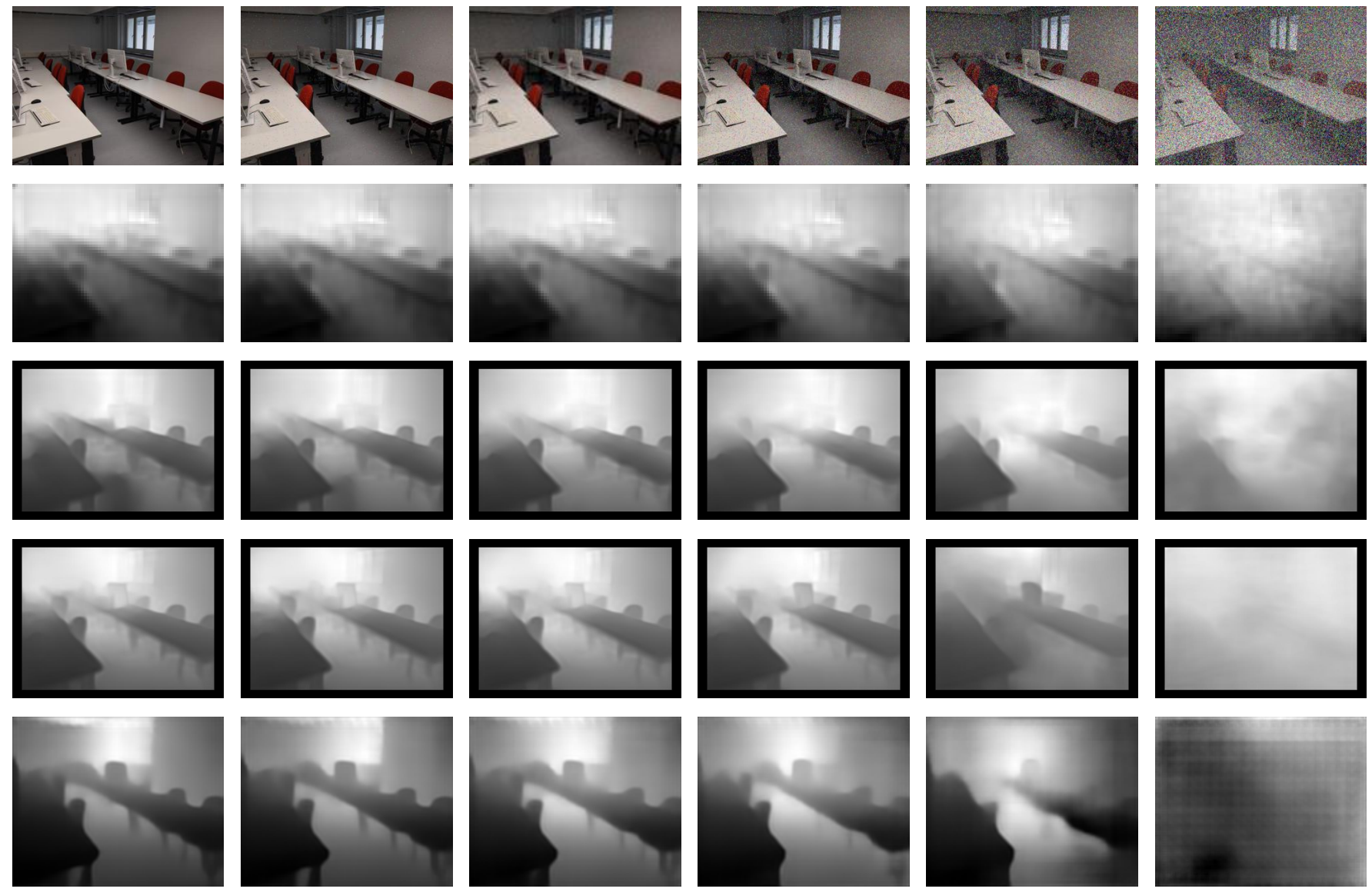

Figure A11: Depth predictions using different SIDE methods on augmented images after adding Salt and Pepper Noise on the original image. Increasing noise level from left to right $(0 \%, 0.5 \%, 1.6 \%, 5 \%, 16 \%, 50 \%)$. From top to bottom: augmented RGB image, Eigen et al. [7], Eigen and Fergus [6] (AlexNet), Eigen and Fergus [6] (VGG), Laina et al. [16] 

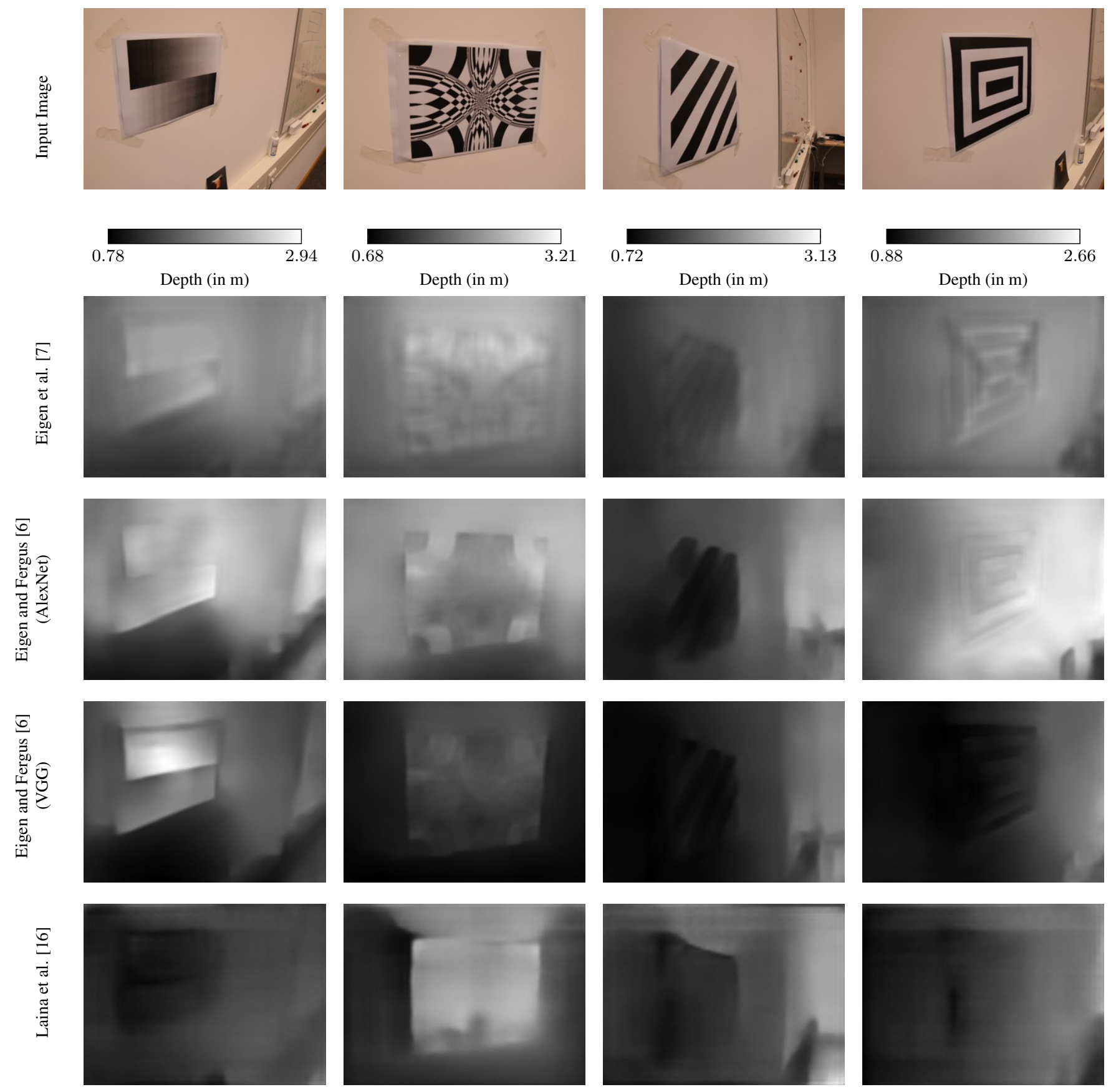

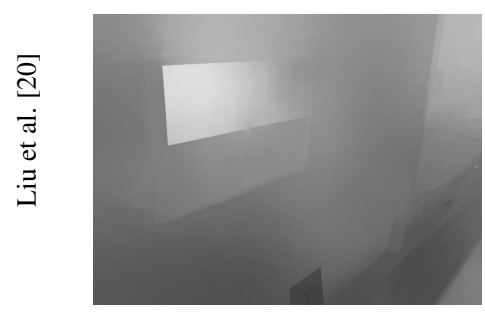

(a) Gradients

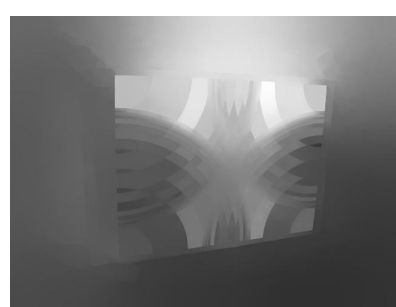

(b) Curves

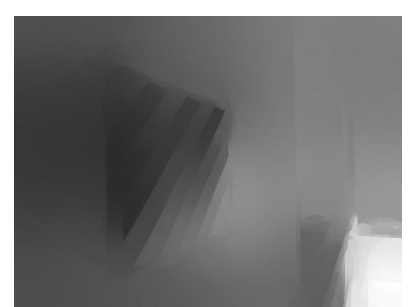

(c) Stripes

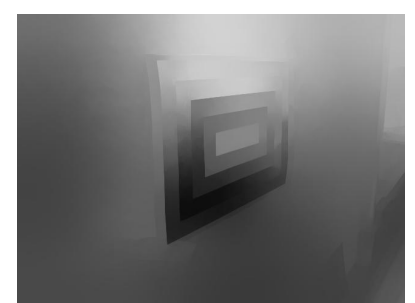

(d) Boxes

Figure A12: Predictions for different printed samples from the Pattern dataset [1] on a planar surface (colums). Predictions using different methods (rows) of the input images (first row). Predicted depth maps are color-coded according to the colormaps shown in the second row 

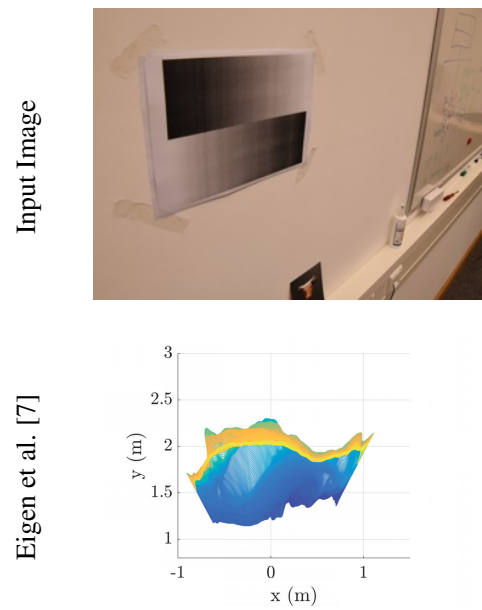

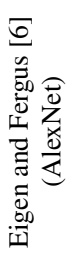

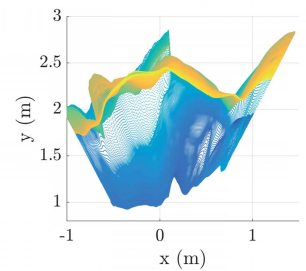

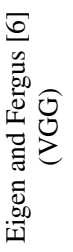
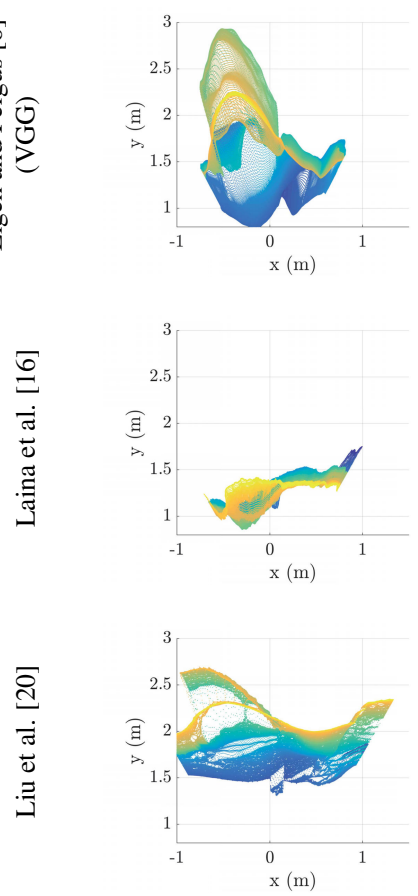

(a) Gradients
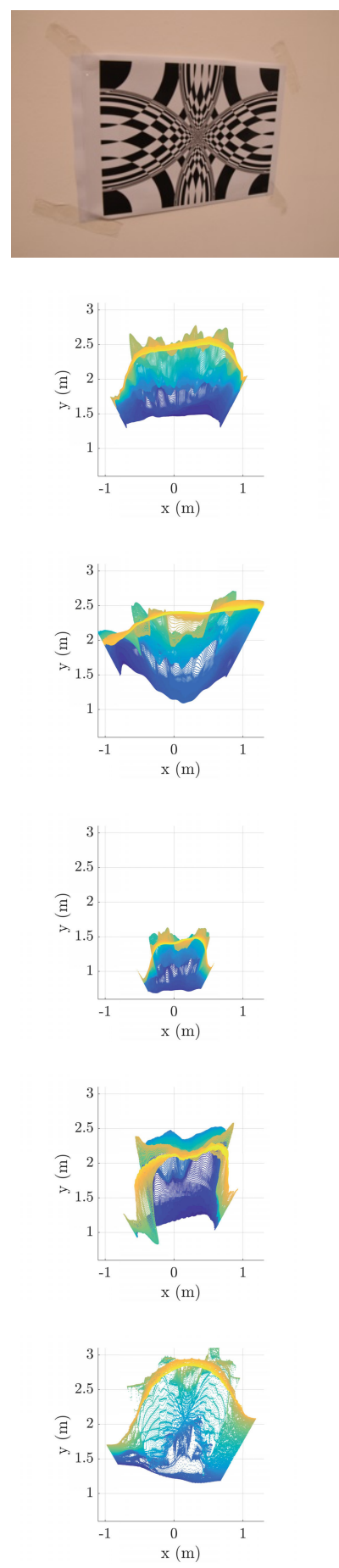

(b) Curves
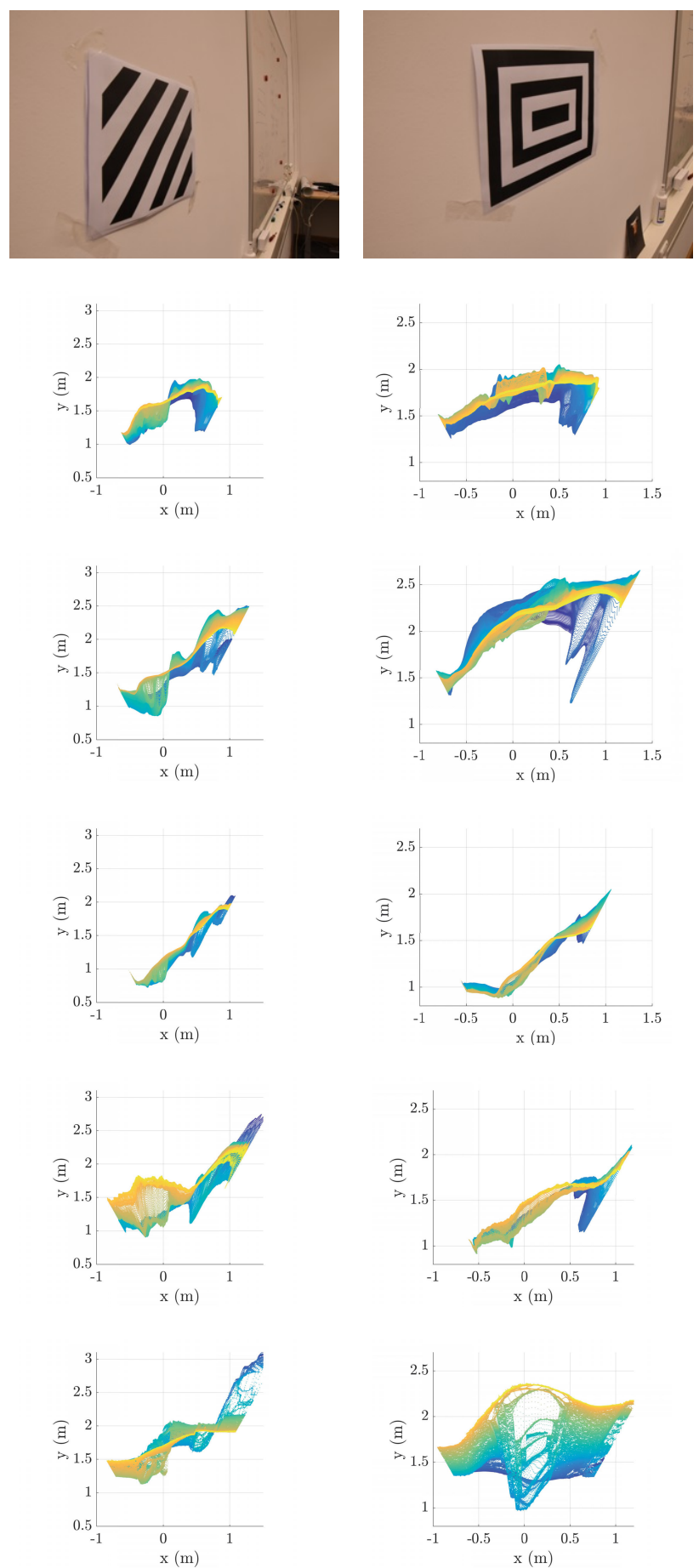

(c) Stripes

(d) Boxes

Figure A13: Top-down views of 3D point clouds for the depth maps in Fig. A12. Each point cloud is presented in a local world coordinate system. Cameras are located in the origin and directed along the y-axis. Colors indicate height in z-direction (from blue to yellow) 